\title{
TOWARDS A NEW FRICTION MODEL FOR SHALLOW WATER EQUATIONS THROUGH AN INTERACTIVE VISCOUS LAYER
}

\author{
François James ${ }^{1, *}$, Pierre-Yves Lagrée ${ }^{2}$, Minh H. LE ${ }^{3}$ and Mathilde Legrand ${ }^{1}$
}

\begin{abstract}
The derivation of shallow water models from Navier-Stokes equations is revisited yielding a class of two-layer shallow water models. An improved velocity profile is proposed, based on the superposition of an inviscid fluid and a viscous layer inspired by the Interactive Boundary Layer interaction used in aeronautics. This leads to a new friction law which depends not only on velocity and depth but also on the variations of velocity and thickness of the viscous layer. The resulting system is an extended shallow water model consisting of three depth-integrated equations: the first two are mass and momentum conservation in which a slight correction on hydrostatic pressure has been made; the third one, known as von Kármán equation, describes the evolution of the viscous layer. This coupled model is shown to be conditionally hyperbolic, and a Godunov-type finite volume scheme is also proposed. Several numerical examples are provided and compared to the Multi-Layer Saint-Venant model. They emphasize the ability of the model to deal with unsteady viscous effects. They illustrate also the phase-lag between friction and topography, and even recover possible reverse flows.
\end{abstract}

Mathematics Subject Classification. 35L60, 35L65, 35Q35, 65M08, 76N17.

Received May 9, 2018. Accepted December 16, 2018.

\section{INTRODUCTION}

Many phenomena in fluvial or maritime hydraulics involve free surface flows in shallow waters for the study e.g. of floods and tides. Shallow water equations were originally introduced by Saint-Venant in 1871 [12] in the context of channel modelling. Since then, the model has been widely extended and is used in the modelling and numerical simulation of a number of natural or man-made phenomena such as river flow [6,27], flood forecasting $[7,36]$, pollutant transport [28,52], dam-break [1,57], tsunami [22,35,49], overland flow $[14,15,18,56]$, soil erosion $[8,43]$ and many others.

The shallow water system can be derived from the incompressible Navier-Stokes equations under several hypotheses; the main one being the long wave approximation meaning that the characteristic wavelength is much larger than the water depth (see Fig. 1 for a sketch and definitions). Two consequences follow then: the hydrostatic pressure law holds, and the viscous term vanishes in the horizontal direction. Next, to proceed from

Keywords and phrases. Shallow water, viscous layer, friction law, Prandtl equation, von Kármán equation.

1 Institut Denis Poisson, Université d'Orléans, Université de Tours, CNRS UMR 7013, Route de Chartres, BP 6759 , 45067 Orléans, France.

2 Institut Jean Le Rond d'Alembert, Sorbonne Université, CNRS, UMR 7190, 75005 Paris, France.

3 Laboratoire d'Hydraulique Saint-Venant - ENPC, CEREMA, EDF R\&D, 78401 Chatou, France.

*Corresponding author: james@math.cnrs.fr

(C) The authors. Published by EDP Sciences, SMAI 2019

This is an Open Access article distributed under the terms of the Creative Commons Attribution License (http://creativecommons.org/licenses/by/4.0), which permits unrestricted use, distribution, and reproduction in any medium, provided the original work is properly cited. 
Navier-Stokes to shallow water, the equations are integrated along the vertical direction. At this point, care has to be taken of the vertical velocity profile, which on the one hand has to be approximated to deal with nonlinearities of the momentum flux, but on the other hand drives the bottom boundary condition, hence the friction phenomena.

Two classical assumptions on the longitudinal velocity profile along the vertical direction lead to explicit integrations. The first one is a viscous Poiseuille-like (i.e. parabolic) profile on the whole water depth which gives rise to a linear (with respect to the depth-averaged velocity) friction term, sometimes referred to as laminar friction. The second one is a constant profile, somehow corresponding to an inviscid fluid; but, by construction, there is a priori no friction term in the integrated equations. Friction has to be added afterwards using empirical laws such as Manning, Chézy, etc (see e.g. [11]). The main drawback of these classical approaches is the nonadaptability of the friction terms for large variations of velocity because the assumed profiles - parabolic or flat - do not hold.

We intend here to pay a particular attention to the fact that these empirical laws are unable to describe the fluid inertia effect or more precisely to predict the phase-lag between the bottom friction and a perturbation of the bed which is known as an essential mechanism for dune or ripple formation [33]. Indeed, it is well reported that for the case of a sub-critical flow on a bump, the maximum of the friction must be slightly shifted upstream of the crest to drag the particles from the troughs up to the crest. Consequently, coupling classical shallow water equations with a mass conservation equation for sediment transport, e.g. Exner equation [19] for bedload case, cannot predict the bed instability, see [10,37] for more details. We look for a more flexible model with a better understanding of how the no-slip boundary condition gives rise to the friction term in the depth-integrated equations. This will allow to recover this phase-lag phenomenon as well as boundary layer separation - a manifestation of the re-circulation of the flow near bottom.

This is done by using an asymptotic description of the fluid as a superposition of an inviscid fluid over a viscous layer located at the bottom, with a strong interaction between the layers. Integrating incompressibility and momentum equations over the water depth leads to a system of two equations being similar in structure with the usual shallow water system, but involving several additional unknown functions related to the viscous layer.

The next step consists therefore in a careful analysis of the viscous layer, whose order of magnitude is quantified by a small parameter $\bar{\delta}$ related to the inverse of the Reynolds number of the flow. Following a classical methodology in aerodynamics, see e.g. [53], we integrate the Prandtl equation along the vertical axis to obtain the so-called von Kármán equation. It describes the evolution of the so-called displacement thickness $\delta_{1}$ (see Fig. 1), which can be interpreted as some physical thickness of the viscous layer. In the case of an inviscid fluid $(\bar{\delta}=0)$, the proposed model degenerates, of course, into the classical shallow water one.

The outline of the paper is as follows. The next two sections contain quite classical material, which is reminded for self-consistency and the reader's convenience. In Section 2 we recall the Navier-Stokes system, and state the long wave approximation which is convenient for shallow water flows. Next, in Section 3 we turn to the viscous layer analysis and derive the Prandtl and von Kármán equations. Appropriate velocity profiles are also introduced. The fourth section is devoted to the derivation of various formulations of the Extended Shallow Water System, which is the main goal of this work. In the next sections, we work only on one of these formulations, namely (4.11), which involves an Interactive Boundary Layer, and can be interpreted as an ideal fluid with a transpiration boudary condition. In Section 5 we study the hyperbolicity of this model, and propose an adaptated finite volume scheme. Finally, in Section 6, we investigate numerically several properties of the model. In particular we evidence the phase-lag effect for flows over short bumps. Furthermore, we compare the present model to the Multi-Layer Saint Venant model proposed in [2]. 


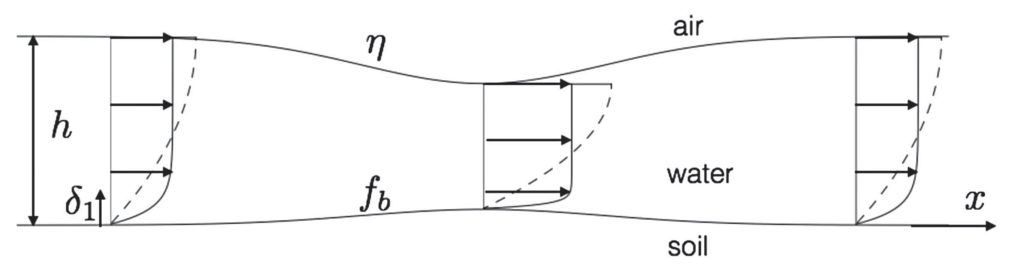

FiguRE 1. Domain under consideration: the water layer is defined by the depth $h(t, x)$ of characteristic value $h_{0}$, the bottom is a given function $f_{b}(x)$ of characteristic horizontal length $L$ and $\eta(t, x)$ is the free surface. Two families of velocity profiles are displayed for the flow over the topography, first with the usual half-Poiseuille description (dashed), and second with the flat profile with a boundary layer (plain). Note that the shear (slope of the velocity at the wall) is completely different in those two descriptions even flux and depth are the same.

\section{From Navier-Stokes to Shallow WATER Equations}

In this section we recall how classical models for shallow waters are obtained from Navier-Stokes equations. The first assumption is a long wave approximation, stating that indeed we deal with a thin layer of water. Next, we integrate along the vertical direction, assuming a given velocity profile on the whole water depth.

\subsection{Navier-Stokes equations}

We consider a fluid evolving in a time-dependant domain $\Omega_{t}=\mathbb{R} \times\left\{f_{b}(x) \leq y \leq \eta(t, x)\right\}$, limited below by a fixed bottom $y=f_{b}(x)$ and above by the free surface $y=\eta(t, x)$. In this study, the properties of the air above the free surface are completely neglected (see Fig. 1).

We start from the dimensionless Navier-Stokes equations, which express the mass and momentum conservations of an incompressible Newtonian fluid [53]. For the sake of simplicity, we limit ourselves in this work to laminar flows. Indeed, the asymptotic analysis of the Navier-Stokes equations is clearer and the resulting description is quantitative. A similar study for turbulent flows can be made with a modified Reynolds tensor. The dimensionless Navier-Stokes equations write

$$
\begin{aligned}
\partial_{x} u+\partial_{y} v & =0 \\
\partial_{t} u+u \partial_{x} u+v \partial_{y} u & =-\partial_{x} p+\frac{1}{\operatorname{Re}} \nabla^{2} u \\
\partial_{t} v+u \partial_{x} v+v \partial_{y} v & =-\partial_{y} p-\frac{1}{\mathrm{Fr}^{2}}+\frac{1}{\operatorname{Re}} \nabla^{2} v,
\end{aligned}
$$

where $u, v$ are the horizontal and vertical velocities respectively and $p$ is the pressure. In these equations, the Reynolds number Re and the Froude number Fr are defined using a reference velocity $u_{0}$ and the same reference length, the depth $h_{0}$ :

$$
\operatorname{Re}=\frac{u_{0} h_{0}}{\nu}, \quad \operatorname{Fr}=\frac{u_{0}}{\sqrt{g h_{0}}},
$$

where $\nu$ is the kinematic viscosity and $g$ is the gravity constant. The Reynolds number expresses the ratio between the inertia force and the viscosity while the Froude number represents the ratio between the kinetic and potential energies.

The system is complemented with the following boundary conditions:

- at the bottom $y=f_{b}(x)$ :

- no-slip condition: $u=v=0$,

- at the free surface $y=\eta(t, x)$ :

- kinematic boundary condition: $v=\partial_{t} \eta+u \partial_{x} \eta$ 
- continuity of the stress tensor: $\sigma \cdot \mathbf{n}=0$, where $\sigma=\left(\begin{array}{cc}2 \partial_{x} u-p & \partial_{x} v+\partial_{y} u \\ \partial_{y} u+\partial_{x} v & 2 \partial_{y} v-p\end{array}\right)$ is the stress tensor and $\mathbf{n}=\frac{1}{\sqrt{1+\left(\partial_{x} \eta\right)^{2}}}\left(\begin{array}{c}\partial_{x} \eta \\ -1\end{array}\right)$ is the outer unit normal to the free surface.

\subsection{Long wave approximation}

Up to now, no hypothesis has been taken into account for the size order of the characteristic quantities $h_{0}, u_{0}$. We have in mind applications to rivers or coastal flows for which the following conditions may be observed: (i) the Reynolds number is large, (ii) the horizontal velocity has small variation along the vertical, (iii) the vertical velocity is small compared to the horizontal velocity.

We introduce the aspect ratio

$$
\varepsilon=\frac{h_{0}}{L} \ll 1,
$$

where $L$ is a characteristic wave length. The long wave scaling consists in assuming that the characteristic horizontal scale is much larger than the vertical one, but also that the characteristic time scale is large. Thus we use the following variable scaling:

$$
x=\frac{\tilde{x}}{\varepsilon}, \quad y=\tilde{y}, \quad t=\frac{\tilde{t}}{\varepsilon}, \quad u=\tilde{u}, \quad v=\varepsilon \tilde{v}, \quad p=\tilde{p} .
$$

Furthermore, since $f_{b}(x)=\tilde{f}_{b}(\tilde{x})$ we have $f_{b}^{\prime}(x)=\varepsilon \tilde{f}_{b}{ }^{\prime}(\tilde{x})$ and so the bottom slope needs to be small enough. The continuity of the stress tensor at the free surface becomes

$$
\left(\begin{array}{l}
\varepsilon\left(\left(2 \varepsilon \partial_{\tilde{x}} \tilde{u}-\tilde{p}\right) \partial_{\tilde{x}} \tilde{\eta}-\varepsilon \partial_{\tilde{x}} \tilde{v}\right)-\partial_{\tilde{y}} \tilde{u} \\
\varepsilon\left(\partial_{\tilde{x}} \tilde{\eta}\left(\partial_{\tilde{y}} \tilde{u}+\varepsilon \partial_{\tilde{x}} \tilde{v}\right)-2 \partial_{\tilde{y}} \tilde{v}\right)-\tilde{p}
\end{array}\right)=\left(\begin{array}{l}
0 \\
0
\end{array}\right) \quad \text { at } \quad \tilde{y}=\tilde{\eta}
$$

Taking an approximation at order $O(\varepsilon)$ yields the following system, sometimes called the RNSP equations (Reduced Navier-Stokes/Prandtl [41]). It is convenient to define an effective Reynolds number, $\mathrm{Re}_{h}$, which takes into account the aspect ratio of the model

$$
\operatorname{Re}_{h}=\varepsilon \operatorname{Re} .
$$

Dropping all the tildes from the variables and unknowns, the system can be written:

$$
\begin{aligned}
\partial_{x} u+\partial_{y} v & =0, \\
\partial_{t} u+u \partial_{x} u+v \partial_{y} u & =-\partial_{x} p+\frac{1}{\operatorname{Re}_{h}} \partial_{y}^{2} u, \\
\partial_{y} p & =-\frac{1}{\operatorname{Fr}^{2}}, \\
\partial_{t} \eta+u \partial_{x} \eta-v & =0 \quad \text { at } \quad y=\eta, \\
p=0, \quad \partial_{y} u & =0 \quad \text { at } \quad y=\eta, \\
u=v & =0 \text { at } y=f_{b} .
\end{aligned}
$$

Therefore, the long wave approximation amounts to neglect the viscosity in horizontal direction (2.5), and to use a hydrostatic pressure law (2.6) as well as a simplified version of the stress tensor continuity at the free surface (2.8). This will be our reference system for the remaining of this article.

In the limit $\operatorname{Re}_{h} \rightarrow \infty$, the case of an incompressible inviscid fluid, the RNSP equations degenerate to the hydrostatic Euler system. Indeed, the equations of mass conservation (2.4), hydrostatic pressure (2.6) and boundary conditions (2.7) and (2.8) at free surface are unchanged, but the momentum conservation equation is replaced by

$$
\partial_{t} u+u \partial_{x} u+v \partial_{y} u=-\partial_{x} p .
$$


Moreover, the hydrostatic Euler system admits a particular solution for which the longitudinal velocity does not depend on $y: u=u_{e}(t, x)$. Thus the momentum equation (2.10) becomes

$$
\partial_{t} u_{e}+u_{e} \partial_{x} u_{e}=-\partial_{x} p
$$

As it is well-known in Inviscid Fluid theory, see e.g. [31], the no-slip boundary condition (2.9) at the bottom is no longer relevant and has to be replaced by a weaker one called the non-penetration condition

$$
\left.v\right|_{y=f_{b}}=u_{e} f_{b}{ }^{\prime} .
$$

Recovering some connection between this solution of inviscid fluid equations and the no-slip condition is precisely the aim of the viscous layer theory, which we will present in Section 4.

\subsection{Classical shallow water equations}

The water depth $h$ and the depth-averaged horizontal velocity $U$ are defined by

$$
h=\eta-f_{b}, \quad h U=\int_{f_{b}}^{\eta} u \mathrm{~d} y .
$$

The shallow water model is derived from the RNSP system by integrating over the whole water depth, from $f_{b}$ to $\eta$, the mass and momentum equations (2.4) and (2.5) together with conditions (2.6)-(2.9). This yields

$$
\begin{aligned}
\partial_{t} h+\partial_{x}(h U) & =0, \\
\partial_{t}(h U)+\partial_{x}\left(\beta h U^{2}\right) & =-h \partial_{x} p-\tau_{b},
\end{aligned}
$$

in which we have introduced the so-called Boussinesq coefficient $\beta$ and the bottom shear stress $\tau_{b}$, also called friction, defined by

$$
\int_{f_{b}}^{\eta} u^{2} d y=\beta h U^{2}, \quad \tau_{b}=\left.\frac{1}{\operatorname{Re}_{h}} \partial_{y} u\right|_{y=f_{b}} .
$$

Notice that $\beta \geq 1$ since, by definition (2.16), we can write

$$
\beta=1+\frac{1}{h} \int_{f_{b}}^{\eta}\left(1-\frac{u}{U}\right)^{2} \mathrm{~d} y
$$

Without complementary equations, a closure relation on the velocity profile is needed in order to compute both the Boussinesq coefficient and the friction term in terms of the conservative variables $(h, h U)$. Let us recall two constitutive profiles which are often adopted in the context of shallow water flows.

Flat profile. This is the most classical approach in hydraulic river modelling. Scaling analysis reveals that the velocity profile is quasi-flat except within a very thin-layer close to the river bed. Based on this consideration, the velocity profile can be assumed to be flat over the whole water depth, so that $\beta$ equals one. The friction term $\tau_{b}$ is not properly defined in this context. The flow can be described using the Euler system resulting in an inviscid shallow water model - equation (2.15) without the friction term $\tau_{b}$.

A large family of empirical friction laws exist, which express the friction as a quadratic function of $U$ with a friction coefficient $C_{f}=O\left(\mathrm{Re}^{-1 / 4}\right)$ for a smooth bottom, see [53]. This coefficient depends on $h$ and $U$ as well, for instance with Chézy, Manning laws, see [11] for a bibliographical study. In summary, this kind of model consists in writing

$$
\beta=1, \quad \tau_{b}=\frac{1}{2} C_{f} U^{2} .
$$

We emphasize again that the friction term derives here from empirical considerations. 
Poiseuille profile. This type of profile is inspired from an analytic solution of the RNSP equations in the case of an uniform flow on a negative constant slope. The balance between the friction and the driving force of the slope gives a self-similar parabolic solution, also known as the half-Poiseuille or Nusselt solution:

$$
\frac{u}{U}=3\left(\zeta-\frac{1}{2} \zeta^{2}\right), \quad 0 \leq \zeta=\frac{y-f_{b}}{h} \leq 1
$$

This choice of profile leads to

$$
\beta=\frac{6}{5}, \quad \tau_{b}=\frac{3}{\operatorname{Re}_{h}} \frac{U}{h} .
$$

The friction is indeed linear with respect to the mean velocity, and is referred to as laminar friction.

Using such a prescribed profile in shallow water equations leads to some important restriction of the model, in particular when dealing with large variations of the velocity. For example it has been reported in [30] that a constant value for the Boussinesq coefficient is less adapted to describe the dynamics of the fluid layer close to a dry-wet transition.

We conclude this section by evidencing that both these models do not present a phase-lag for the flow over a bump. Let us study a steady linearized solution of the usual shallow water equations. Consider such a small perturbation of the bed $f_{b}$ that we can write in the form $f_{b}=\varepsilon f_{b}^{1}$, where $\varepsilon$ is just a small parameter and not necessarily the aspect ratio defined before. We look for the solution in the form

$$
h=h^{0}+\varepsilon h^{1}, \quad U=U^{0}+\varepsilon U^{1} .
$$

For high Reynolds numbers, we see from relations (2.18) and (2.19) that the friction is negligible. We can consider therefore $h, U$ solution of the following frictionless and stationary shallow water equations

$$
\partial_{x}(h U)=0, \quad \partial_{x}\left(\beta h U^{2}+\frac{1}{2 \mathrm{Fr}^{2}} h^{2}\right)=-\frac{1}{\operatorname{Fr}^{2}} h f_{b}^{\prime} .
$$

Inserting (2.20) in (2.21) and identifying powers of $\varepsilon$ leads to a cascade of equations for each terms $h^{i}, U^{i}, i=$ 0,1 . It is easily checked that the zeroth-order terms $h^{0}$ and $U^{0}$ are constant. For the first-order terms, a straightforward calculation leads to

$$
h^{0} \partial_{x} U^{1}+U^{0} \partial_{x} h^{1}=0, \quad\left(\frac{h^{0}}{\operatorname{Fr}^{2}}-\beta\left(U^{0}\right)^{2}\right) \partial_{x} h^{1}=-\frac{h^{0}}{\operatorname{Fr}^{2}}\left(f_{b}^{1}\right)^{\prime} .
$$

By introducing the local Froude number, $\mathrm{Fr}_{0}$, we can express the linearized solution in the form

$$
\operatorname{Fr}_{0}^{2}=\frac{\beta\left(U^{0}\right)^{2}}{h^{0}} \operatorname{Fr}^{2}, \quad h=h^{0}+\frac{1}{\operatorname{Fr}_{0}^{2}-1} f_{b}, \quad U=U^{0}+\frac{U^{0}}{h^{0}} \frac{1}{1-\operatorname{Fr}_{0}^{2}} f_{b} .
$$

As we can see, $U$ is exactly in-phase with $f_{b}$. As a consequence, local maxima of the friction estimated by empirical formulas (2.18) or (2.19) are always reached at $f_{b}^{\prime}=0$, that is at the top of the bump. Indeed, because $\partial_{x} h=\partial_{x} U=0$ if $f_{b}^{\prime}=0$ by $(2.22)$, it follows that $\partial_{x} \tau_{b}=\partial_{h} \tau_{b} \partial_{x} h+\partial_{U} \tau_{b} \partial_{x} U=0$.

\section{VisCOUS LAYER ANALYSIS}

We turn now to the main step towards the model we look for. It mainly consists in dividing the fluid in two layers:

- an inviscid fluid layer dealing with the free surface;

- a thin viscous layer with the no-slip condition at the bottom. 
In the first layer addressing to inviscid fluid, we take advantage of the explicit integration along the vertical. In the second one describing viscous layer, we take into account the viscosity in the vertical direction and recover some friction in the integrated equations. This section is devoted to the study of the viscous layer, and to the analysis of the interactions between the two layers.

We introduce a small parameter $\bar{\delta}$, whose magnitude will be specified below. It is related to the thickness of the viscous layer, but does not correspond to its actual physical value. We follow the classical strategy used in the boundary layer theory $[50,53]$ except that in that case $\bar{\delta} \rightarrow 0$, whereas we keep a finite value here. The first step is to rescale again the RNSP equations with the thin layer scaling to obtain a set of the well-known Prandtl equations. The next step consists in vertical integration of these equations over the viscous layer. This leads to the so-called von Kármán equation, where extra unknowns are introduced. Finally, some suitable assumptions have to be made on the velocity profile in order to obtain a closed model.

\subsection{Prandtl equations}

We introduce the following change of variables, referred to as the Prandtl shift:

$$
x=\bar{x}, \quad y=\bar{\delta} \bar{y}+f_{b}, \quad t=\bar{t}, \quad p=\bar{p}, \quad u=\bar{u}, \quad v=\bar{\delta} \bar{v}+f_{b}^{\prime} u .
$$

By this, the RNSP equations (2.4)-(2.6) and (2.9) are transformed into a set of boundary layer equations on a flat bottom. In particular, the momentum equation reads

$$
\partial_{\bar{t}} \bar{u}+\bar{u} \partial_{\bar{x}} \bar{u}+\bar{v} \partial_{\bar{y}} \bar{u}=-\partial_{\bar{x}} \bar{p}+\frac{f_{b}^{\prime}}{\bar{\delta}} \partial_{\bar{y}} \bar{p}+\frac{1}{\bar{\delta}^{2} \operatorname{Re}_{h}} \partial_{\bar{y}}^{2} \bar{u} .
$$

There are several possible scalings for $\bar{\delta}$ in terms of $\operatorname{Re}_{h}$ :

- if $\bar{\delta}$ verifies $\bar{\delta}^{2} \operatorname{Re}_{h} \gg 1$, we recover the inviscid fluid equations;

- if $\bar{\delta}$ satisfies $\bar{\delta}^{2} \operatorname{Re}_{h} \ll 1$, we obtain $\partial_{\bar{y}}^{2} \bar{u}=0$ that leads to $\bar{u}=0$ due to the continuity of the stress tensor and the no-slip condition. So we do not consider this trivial case;

- the last possibility is $\bar{\delta}^{2} \operatorname{Re}_{h} \sim 1$, which balances the convective terms and the diffusive one. It is called dominant balance or least degeneracy principle [58], and allows to preserve as many as possible terms in the equations.

This is why in what follows, we consider the scaling

$$
\bar{\delta}=\frac{1}{\sqrt{\mathrm{Re}_{h}}} \ll 1 .
$$

With this choice of $\bar{\delta}$, the viscous term appears with the same order as the other terms in the momentum equation. We obtain the Prandtl equations written in viscous layer variables:

$$
\left\{\begin{array}{l}
\partial_{\bar{x}} \bar{u}+\partial_{\bar{y}} \bar{v}=0 \\
\partial_{\bar{t}} \bar{u}+\bar{u} \partial_{\bar{x}} \bar{u}+\bar{v} \partial_{\bar{y}} \bar{u}=-\partial_{\bar{x}} \bar{p}-\frac{f_{b}^{\prime}}{\operatorname{Fr}^{2}}+\partial_{\bar{y}}^{2} \bar{u} \\
\partial_{\bar{y}} \bar{p}=-\frac{\bar{\delta}}{\operatorname{Fr}^{2}} \\
\bar{u}=\bar{v}=0 \quad \text { at } \bar{y}=0 .
\end{array}\right.
$$

We notice that this system of equations is in the same form as the Prandtl equations obtained directly from Navier-Stokes equations with classical boundary layer scaling ([53], Ch. VII) except for the topography term in the momentum equation (3.4). 


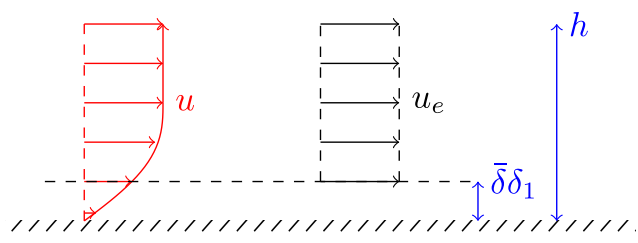

FiguRE 2. Interpretation of the displacement thickness, the flux of mass is the same in the viscous layer and in a equivalent layer of inviscid fluid shifted by an amount of $\bar{\delta} \delta_{1}$.

Up to now, we do not have enough boundary conditions for the viscous layer. The natural connection consists in assuming that the velocity at the "top" of the viscous layer coincides with the velocity of inviscid layer. Precisely, we impose the following matching boundary condition

$$
\bar{u}(\bar{t}, \bar{x}, \bar{\eta})=u_{e}(t, x), \quad \text { where } \quad \bar{\eta}=\frac{\eta-f_{b}}{\bar{\delta}},
$$

which is obviously compatible with the Prandtl shift (3.1) since $\bar{x}=x$ and $\bar{t}=t$. Notice that in classical boundary layer theory the limit is given by the asymptotic matching $\bar{u}(\bar{t}, \bar{x}, \bar{y} \rightarrow \infty) \rightarrow u_{e}(t, x)$.

\subsection{Von Kármán equation}

The von Kármán equation expresses the defect of velocity between the inviscid fluid and the viscous layer. A classical way to obtain such an equation consists in writing the Prandtl equation, then introducing the velocity defect $\left(u_{e}-\bar{u}\right.$ ), and finally integrating it on the viscous layer (see Schlichting [53]). We introduce the following two integrated quantities, see Figure 2:

Definition 3.1. Let $U$ be the depth-averaged velocity. We define

- the displacement thickness $\delta_{1}$ given by

$$
h U=\left(h-\bar{\delta} \delta_{1}\right) u_{e}
$$

- the momentum thickness $\delta_{2}$ given by

$$
\int_{f_{b}}^{\eta} u^{2} \mathrm{~d} y=\left(h-\bar{\delta}\left(\delta_{1}+\delta_{2}\right)\right) u_{e}^{2}
$$

Physically, the displacement thickness expresses the distance by which the ground should be displaced to obtain an inviscid fluid with velocity $u_{e}$ and the same flow rate $h U$ (see Fig. 2). In the same way, the momentum thickness accounts for the loss of momentum in the viscous layer.

A simple computation from (3.8) and (3.9) leads to the following expressions for these quantities:

$$
\delta_{1}=\int_{0}^{\bar{\eta}}\left(1-\frac{\bar{u}}{u_{e}}\right) \mathrm{d} \bar{y}, \quad \delta_{2}=\int_{0}^{\bar{\eta}} \frac{\bar{u}}{u_{e}}\left(1-\frac{\bar{u}}{u_{e}}\right) \mathrm{d} \bar{y} .
$$

In the limit $\bar{\delta} \rightarrow 0$, we recover the classical formulæ for $\delta_{1}, \delta_{2}$ in the boundary layer scaling [53]:

$$
\delta_{1}=\int_{0}^{+\infty}\left(1-\frac{\bar{u}}{u_{e}}\right) \mathrm{d} \bar{y}, \quad \delta_{2}=\int_{0}^{+\infty} \frac{\bar{u}}{u_{e}}\left(1-\frac{\bar{u}}{u_{e}}\right) \mathrm{d} \bar{y}
$$

Proposition 3.2. The evolution of the displacement and momentum thicknesses are ruled by the so-called von Kármán equation:

$$
\partial_{t}\left(u_{e} \delta_{1}\right)+u_{e} \delta_{1} \partial_{x} u_{e}+\partial_{x}\left(u_{e}^{2} \delta_{2}\right)=\bar{\tau}_{b},
$$


where $\bar{\tau}_{b}$ denotes the parietal constraints:

$$
\bar{\tau}_{b}=\left.\partial_{\bar{y}} \bar{u}\right|_{\bar{y}=0}=\frac{\tau_{b}}{\bar{\delta}} .
$$

Proof. First we notice that the Prandtl shift leads to following relations

$$
\partial_{x}=\partial_{\bar{x}}-\frac{f_{b}^{\prime}}{\bar{\delta}} \partial_{\bar{y}}, \quad \partial_{y}=\frac{1}{\bar{\delta}} \partial_{\bar{y}}
$$

The momentum equation (2.11) for inviscid flow can be rewritten as

$$
\partial_{\bar{t}} \bar{u}_{e}+\bar{u}_{e} \partial_{\bar{x}} \bar{u}_{e}=-\partial_{\bar{x}} \bar{p}+\frac{f_{b}^{\prime}}{\bar{\delta}} \partial_{\bar{y}} \bar{p}=-\partial_{\bar{x}} \bar{p}-\frac{f_{b}^{\prime}}{\mathrm{Fr}^{2}},
$$

in which we have used (3.5) to rewrite the right-hand side. The difference between this equation and (3.4) gives

$$
\partial_{\bar{t}}\left(\bar{u}_{e}-\bar{u}\right)+\bar{u}_{e} \partial_{\bar{x}} \bar{u}_{e}-\bar{u} \partial_{\bar{x}} \bar{u}-\bar{v} \partial_{\bar{y}} \bar{u}=-\partial_{\bar{y}}^{2} \bar{u} .
$$

Through (3.3) and (3.6), we can rearrange the term $\bar{v}=-\int_{0}^{\bar{y}} \partial_{\bar{x}} \bar{u}$. Furthermore we get

$$
\partial_{\bar{t}}\left(\bar{u}_{e}-\bar{u}\right)+\left(\bar{u}_{e}-\bar{u}\right) \partial_{\bar{x}} \bar{u}_{e}+\bar{u} \partial_{\bar{x}}\left(\bar{u}_{e}-\bar{u}\right)+\partial_{\bar{y}} \bar{u} \int_{0}^{\bar{y}} \partial_{\bar{x}} \bar{u} \mathrm{~d} \bar{y}=-\partial_{\bar{y}}^{2} \bar{u} .
$$

Using integration by parts, the last term in the left-hand side can be rewritten as

$$
\partial_{\bar{y}} \bar{u} \int_{0}^{\bar{y}} \partial_{\bar{x}} \bar{u} \mathrm{~d} \bar{y}=-\bar{u} \partial_{\bar{x}} \bar{u}+\partial_{\bar{y}}\left(\bar{u} \int_{0}^{\bar{y}} \partial_{\bar{x}} \bar{u} \mathrm{~d} \bar{y}\right) .
$$

Now we integrate the resulting equation over $\bar{y}$, between 0 and $\bar{\eta}$, together with the matching boundary condition (3.7) to obtain the momentum integral equation

$$
\begin{gathered}
\partial_{\bar{t}} \int_{0}^{\bar{\eta}}\left(\bar{u}_{e}-\bar{u}\right) \mathrm{d} \bar{y}+\partial_{\bar{x}} \bar{u}_{e} \int_{0}^{\bar{\eta}}\left(\bar{u}_{e}-\bar{u}\right) \mathrm{d} \bar{y}+\int_{0}^{\bar{\eta}} \bar{u} \partial_{\bar{x}}\left(\bar{u}_{e}-\bar{u}\right) \mathrm{d} \bar{y}-\int_{0}^{\bar{\eta}} \bar{u} \partial_{\bar{x}} \bar{u} \mathrm{~d} \bar{y}+u_{e} \int_{0}^{\bar{\eta}} \partial_{\bar{x}} \bar{u} \mathrm{~d} \bar{y} \\
=\left.\partial_{\bar{y}} \bar{u}\right|_{\bar{y}=0} .
\end{gathered}
$$

The last three terms of the left-hand side are now rewritten as $\partial_{\bar{x}} \int_{0}^{\bar{\eta}} \bar{u}\left(\bar{u}_{e}-\bar{u}\right) \mathrm{d} \bar{y}$. Moreover, since $u_{e}$ is independent of $\bar{y}$, we have the relations

$$
u_{e} \delta_{1}=\int_{0}^{\left(\eta-f_{b}\right) / \delta}\left(u_{e}-\bar{u}\right) \mathrm{d} \bar{y}, \quad u_{e}^{2} \delta_{2}=\int_{0}^{\left(\eta-f_{b}\right) / \delta} \bar{u}\left(u_{e}-\bar{u}\right) \mathrm{d} \bar{y} .
$$

Since $t=\bar{t}$ and $x=\bar{x}$ in the Prandtl shift, and all unknowns in the equation are independent on $\bar{y}$, we can drop the bar symbols in the derivatives. From definition (3.11) we obtain the final form (3.10) of the von Kármán equation. Finally, the relation between the friction $\tau_{b}$ and the rescaled one $\bar{\tau}_{b}$ in (3.11) is an easy consequence of the Prandtl shift and definition (2.16).

Coupled with (2.11) on the velocity $u_{e}$ of inviscid fluid, the von Kármán equation (3.10) gives only a partial representation of the boundary layer, since it involves four additional unknowns: $u_{e}, \delta_{1}, \delta_{2}$, $\bar{\tau}_{b}$. To proceed further towards an integrated model, we have to specify velocity profiles in the viscous layer. 


\subsection{Velocity profile in the viscous layer}

Through the viscous layer, the velocity $\bar{u}$ varies from 0 (at the bottom) to the inviscid fluid velocity $u_{e}$. Therefore we introduce a profile function $\varphi$ as well as a scaling factor $\Delta(\bar{t}, \bar{x})$, chosen in such a way that $\Delta$ quantifies the physical thickness of the viscous layer. Following [53], we wish to have

$$
\frac{\bar{u}(\bar{t}, \bar{x}, \bar{y})}{u_{e}}=\varphi\left(\frac{\bar{y}}{\Delta}\right)=\varphi(\xi), \quad \xi=\frac{\bar{y}}{\Delta(\bar{t}, \bar{x})} .
$$

Therefore we choose $0<\Delta \leq \bar{\eta}$, and a profile function $\varphi(\xi)$ such that

$$
\varphi(0)=0, \quad \varphi(\xi \geq 1)=1, \quad \int_{0}^{1}(1-\varphi) \mathrm{d} \xi=\alpha_{1}<+\infty, \quad \int_{0}^{1} \varphi(1-\varphi) \mathrm{d} \xi=\alpha_{2}<+\infty .
$$

Hence, by definition of $\delta_{1}$ and $\delta_{2}$, we can write

$$
\begin{aligned}
& \delta_{1}=\int_{0}^{\bar{\eta}}\left(1-\frac{\bar{u}}{u_{e}}\right) \mathrm{d} \bar{y}=\Delta \int_{0}^{1}(1-\varphi) \mathrm{d} \xi=\Delta \alpha_{1}, \\
& \delta_{2}=\int_{0}^{\bar{\eta}} \frac{\bar{u}}{u_{e}}\left(1-\frac{\bar{u}}{u_{e}}\right) \mathrm{d} \bar{y}=\Delta \int_{0}^{1} \varphi(1-\varphi) \mathrm{d} \xi=\Delta \alpha_{2} .
\end{aligned}
$$

To link these variables, we introduce the shape factor $H$ which only depends on the profile function $\varphi$

$$
H=\frac{\delta_{1}}{\delta_{2}}=\frac{\int_{0}^{1}(1-\varphi) \mathrm{d} \xi}{\int_{0}^{1} \varphi(1-\varphi) \mathrm{d} \xi} \geq 1 .
$$

The parietal constraints can also be expressed in terms of $\varphi$ and $u_{e}$ as

$$
\bar{\tau}_{b}=\left.\partial_{\bar{y}} \bar{u}\right|_{\bar{y}=0}=\frac{\varphi^{\prime}(0)}{\Delta} u_{e}=\frac{\alpha_{1} \varphi^{\prime}(0)}{\delta_{1}} u_{e}=\frac{f_{2} H}{\delta_{1}} u_{e}
$$

where the parameter $f_{2}$ is known as the friction factor (see [41,53]) and $f_{2} H=\alpha_{1} \varphi^{\prime}(0)$.

At this stage, choosing a velocity profile in the viscous layer amounts to impose a closure formula on the shape factor $H$ and the friction factor $f_{2}$. Several shapes can be used for the profile, including turbulent ones. As far as laminar profiles are concerned, we refer to Chapter X from [53] for elements of comparisons between different profiles. We shall assume that $\varphi$ depends solely on the variable $\xi$ according to the similarity principle [53] on velocity profile over a flat plane at zero-incidence. For the sake of clarity, let us briefly present in what follows several classical profile functions in viscous layer from which we establish some instructive laws on $H$ and $f_{2}$.

Pohlhausen polynomial profile. This kind of approach is known as the Pohlhausen solution which consists in considering a polynomials approximation of the velocity profile. The polynomial coefficients are chosen such that $\varphi(\xi)$ satisfies the boundary conditions (3.13). The simplest case consists of a linear profile. Higher order polynomials can be derived by imposing additional conditions $0=\varphi^{\prime}(1)=\varphi^{\prime \prime}(1)=\cdots$ meaning that the transition between the viscous layer and the inviscid layer must be smooth. For example, the second-order profile writes

$$
\varphi(\xi)=2 \xi-\xi^{2}, \quad H=2.5, \quad f_{2}=0.267 .
$$

Notice that this parabolic profile should not be confused with the Poiseuille profile since their physical meanings are different: the first one is only the velocity profile in viscous layer while the second one is the velocity profile in whole water depth. 

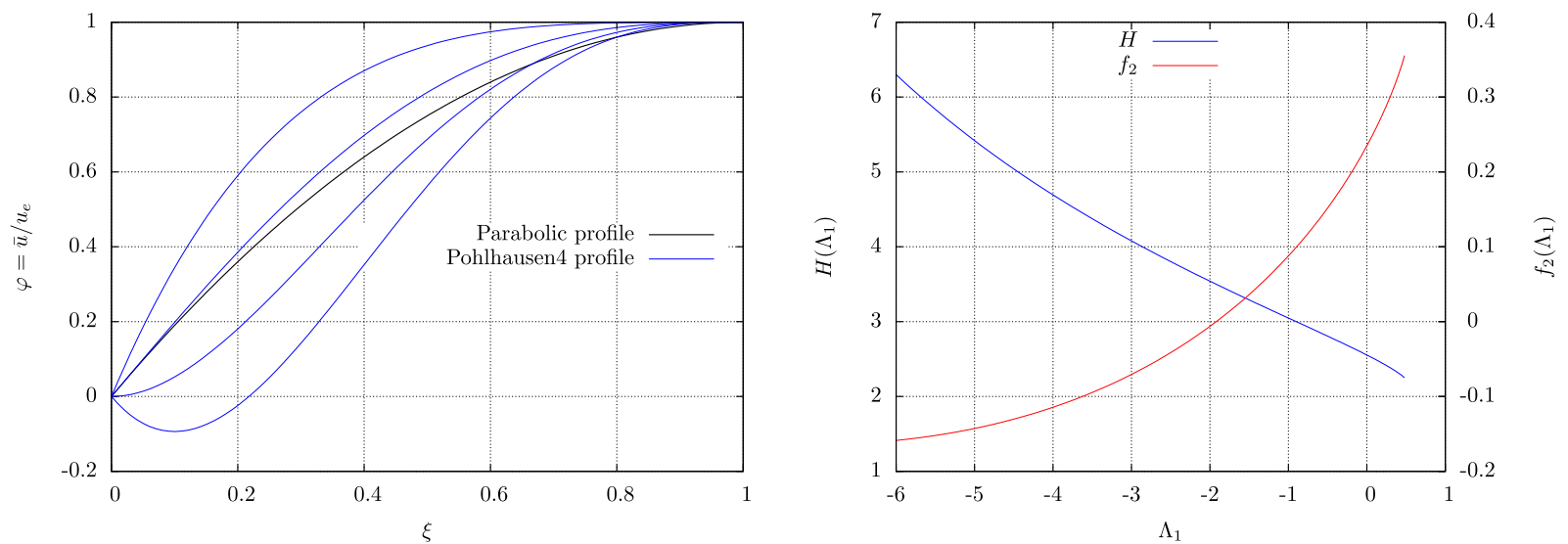

FiguRE 3. Polynomial approximation of the velocity profile: Left panel: parabolic (black) vs. Pohlhausen of order 4 profiles with $\Lambda=12,0,-12,-24$ (blue); Right panel: closure on the shape factor $H$ and and the friction $f_{2}$ factors based on Pohlhausen of order 4. Note that reverse flows $f_{2}<0$ are possible (color online).

Flow separation can be achieved by employing a fourth-order polynomial profile with a free parameter $\Lambda$. The resulting function, named Pohlhausen4 in the following, takes the form

$$
\varphi(\xi)=\left(2 \xi-2 \xi^{3}+\xi^{4}\right)+\frac{\Lambda}{6} \xi(1-\xi)^{3} .
$$

The parameter $\Lambda$ is related to the pressure gradient, and therefore to the variation of velocity of the inviscid flow. Indeed, taking the second-order derivative $\varphi^{\prime \prime}(0)$ and by evaluating Prandtl momentum equation (3.4) at $\bar{y}=0$ we deduce

$$
\Lambda=-\varphi^{\prime \prime}(0)=-\left.\frac{\Delta^{2}}{u_{e}} \partial_{\bar{y}}^{2} \bar{u}\right|_{\bar{y}=0}=-\frac{\Delta^{2}}{u_{e}} \partial_{x} p=\Delta^{2} \partial_{x} u_{e},
$$

where the last equality is the stationary version of the momentum equation (2.10) in the inviscid layer. Since by definition $\varphi(\xi) \leq 1$, we have $\Lambda \leq 12$, and we emphasize that for $\Lambda \leq-12$, the velocity profiles exhibit negative regions that correspond to reverse flow (see Fig. 3, left side).

We turn now to study the shape and friction factors based on the profile under consideration. First, substituting the fourth-order polynomial into definitions (3.14) and (3.15) allows to express $H, f_{2}$ as explicit functions of $\Lambda$, but they are omitted here for the sake of compactness. Nevertheless we notice that these relations are just formal since the physical thickness $\Delta(\bar{t}, \bar{x})$ remains unknown once the velocity $\bar{u}$ in viscous layer has not yet been solved. In practice, it is more convenient to replace $\Delta$ by the displacement thickness $\delta_{1}$. A possible way, according to $[40,41]$, is to introduce a new parameter $\Lambda_{1}$, inspired from the definition of $\Lambda$, given by

$$
\Lambda_{1}=\delta_{1}^{2} \partial_{x} u_{e}=\left(\frac{36-\Lambda}{120}\right)^{2} \Delta^{2} \partial_{x} u_{e}=\left(\frac{36-\Lambda}{120}\right)^{2} \Lambda
$$

in which we have used the relation $\delta_{1} / \Delta=(36-\Lambda) / 120$ obtained by substituting the fourth-order polynomial into definition of $\delta_{1}$. Moreover, equation (3.16) leads to $\Lambda_{1}$ being monotone on the physical range $\Lambda \leq 12$. Consequently, the factors $H, f_{2}$ can also be expressed as functions of $\Lambda_{1}$. We represent on Figure 3 , on the right side, the functions $H\left(\Lambda_{1}\right)$ and $f_{2}\left(\Lambda_{1}\right)$ with $\Lambda$ ranging from -24 to 12 that corresponds to $-6 \leq \Lambda_{1} \leq 0.48$.

Falkner Skan profile. Polynomial profiles, despite their simplicity, are rather artificial. Their construction is based only on some suitable boundary conditions. An alternative approach, that might be more interesting, 

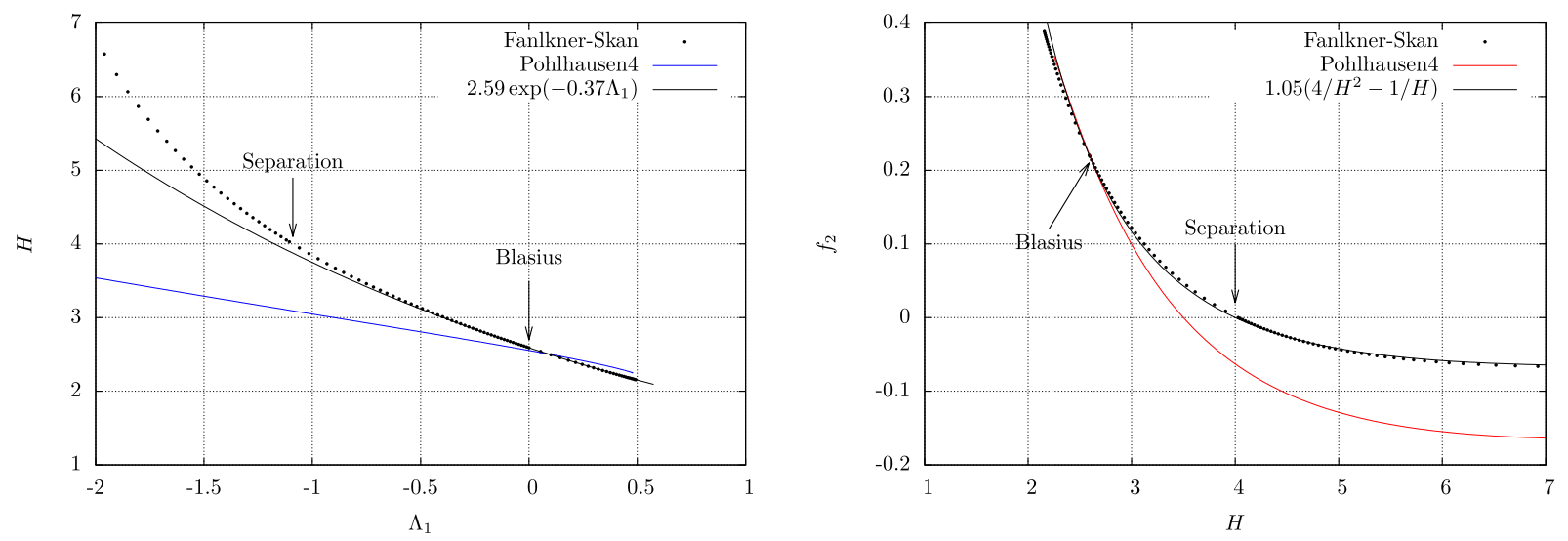

Figure 4. Falkner-Skan vs. Pohlhausen order 4 closures: shape factor $H$ (left panel) as a function of $\Lambda_{1}=\delta_{1}^{2} \partial_{x} u_{e}$ and friction factor $f_{2}$ (right panel) as a function of $H$.

is to use exact solutions of boundary layer equations in order to establish more physical closures. Such an approach can be done by employing the solution to the Falkner-Skan equation [20] - a third-order boundary value problem whose resolution is still complicated (see e.g. $[9,34,60]$ ). It plays an important role to illustrate the main physical features of boundary layer phenomena. This solution describes the form of an external laminar boundary layer of a flow over a wedge. The Blasius solution for a flat plate is a particular case of this solution.

We do not present here any details on the resolution of the Falkner-Skan equation but focus on the construction of closure formulæ and compare the obtained results with those given by Pohlhausen4. First, we solve the Falkner-Skan equation on the whole physical range of pressure gradient, corresponding to the case of accelerated, decelerated and reverse flows, to obtain all the values of the triplet $\left(\Lambda_{1}, H, f_{2}\right)$. Next, we find out a numerical relation between these parameters inspired by the approach presented for Pohlhausen4. On Figure 4, it is found that the Pohlhausen4 closure, although its purely algebraic derivation, presents a good agreement with Falkner-Skan when $\Lambda_{1} \geq 0$ corresponding to accelerated flows. In particular, exact value of Blasius solution $\left(\Lambda_{1}=0, H=2.59, f_{2}=0.22\right)$ is very close to that given by Pohlhausen4 $\left(\Lambda_{1}=0, H=2.554, f_{2}=0.235\right)$. However, these closures diverge in regions of decelerated and reverse flows $\left(\Lambda_{1}<0\right)$. Incipient separation $\left(f_{2}=0\right)$ is reached at $\left(\Lambda_{1}=-1.09, H=4\right)$ while it is $\left(\Lambda_{1}=-1.92, H=3.5\right)$ for Pohlhausen of order 4. Finally, a value $\left(\Lambda_{1}=0.6, H=2.074\right)$ is found as limit of physical range of Falkner-Skan solution.

Lagrée and Lorthois [41] proposed the following ad-hoc closure based on a fitting of Falkner-Skan solution

$$
H=\left\{\begin{array}{ll}
2.59 \mathrm{e}^{-0.37 \Lambda_{1}} & \text { if } \Lambda_{1}<0.6, \\
2.074 & \text { otherwise, }
\end{array} \text { and } f_{2}=1.05\left(\frac{4}{H^{2}}-\frac{1}{H}\right) .\right.
$$

As we can see on Figure 4, this numerical law presents a good agreement near Blasius solution (both accelerated and decelerated flows). These regions are also the most concerned cases in river hydraulic application (i.e. with small bed perturbation). Especially, when plotting $f_{2}$ as function of $H$, an excellent agreement is found. This is why we adopt (3.17) for our numerical study of the present model.

Finally, it is important to emphasize that both polynomial and numerical closure for $H$ and $f_{2}$ are based on steady solutions of the boundary layer equations.

\section{TOWARDS EXTENDED SHALLOW WATER MODELS}

Once an appropriate closure for the velocity profile in the viscous layer is provided, such as presented above, the extended model consists in coupling the system of depth-integrated equations (2.14) and (2.15) with the 
von Kármán equation (3.10). Compared to previous derivations of the shallow water model, e.g. [23], we have noticed, on the one hand, the dependence in $\delta_{1}, \delta_{2}$ of the momentum equation and, on the other hand, the friction term $\tau_{b}$ at order one in $\bar{\delta}$.

In what follows, we start by analyzing the interactions between inviscid and viscous layers through definitions (3.8) and (3.9) for $\delta_{1}$ and $\delta_{2}$, and the von Kármán equation (3.10) which describes their evolution. This analysis leads to several equivalent forms of the coupled system. In particular we obtain on a first hand an apparent topography formulation and on a second hand an Interactive Boundary Layer model. We choose the latter one, system (4.11) below, which is up to now the most tractable one, to illustrate our purpose in Sections 5 and 6 .

\subsection{A first extended model}

We start from the system of depth-integrated equations (2.14)-(2.15) and inviscid fluid equation (2.11). We emphasize that relation (3.9) does not give any closure for the momentum flux, but this expression for the momentum flux is in fact the most convenient one since it takes into account the effect of the viscous layer. We rewrite the momentum equation (2.15) with a generic form of the flux together with relation (3.11) on parietal constraints:

$$
\partial_{t}(h U)+\partial_{x} J=-h \partial_{x} p-\bar{\delta} \bar{\tau}_{b},
$$

where $J=\int_{f_{b}}^{\eta} u^{2} d y$ is the momentum flux for which we seek a closure. We evidence now the fact that a convenient definition of $J$ allows to recover the von Kármán equation from this system of integrated equations. The proof of the following two propositions is given in Appendices A and B.

Proposition 4.1. Let $\left(h, U, u_{e}, J\right)$ be solution to (2.11), (2.14) and (4.1). Assume $\delta_{1}$ is defined by (3.8). Then $\delta_{1}$ and $\delta_{2}$ solve the von Kármán equation (3.10) if and only if there holds

$$
J=\left(h-\bar{\delta}\left(\delta_{1}+\delta_{2}\right)\right) u_{e}^{2}
$$

In the above proposition, we only use the three equations (2.11), (2.14), (4.1) and definition (3.8) of the displacement thickness. Replacing the physical definition (3.8) by the von Kármán equation (3.10) will give back the physical definition in the sense of characteristics as explained in the next proposition.

Proposition 4.2. Let $J$ be defined by (4.2), and $\left(h, U, \delta_{1}, u_{e}\right)$ be a (smooth) solution to the system of equations (2.11), (2.14) and (4.1) together with von Kármán equation (3.10). Denoting by $\delta_{1}^{*}$ the thickness obtained using (3.8), we have

$$
\partial_{t}\left(u_{e}\left(\delta_{1}-\delta_{1}^{*}\right)\right)-u_{e} \partial_{x}\left(u_{e}\left(\delta_{1}-\delta_{1}^{*}\right)\right)=0
$$

In other words the error between these displacement thickness is constant along the characteristics of the inviscid fluid. Hence, if initially $\delta_{1}=\delta_{1}^{*}$ then it is true for all times.

Putting together the results of Proposition 4.1 and the closure formulæ (3.14) and (3.15) on the velocity profile in the viscous layer, we obtain the following expressions for the momentum flux

$$
J=\left(h-\bar{\delta} \delta_{1}\left(1+\frac{1}{H}\right)\right) u_{e}^{2}=h U^{2}+\bar{\delta} \delta_{1}\left(1-\frac{1}{H}-\bar{\delta} \delta_{1} / h\right) u_{e}^{2} .
$$

The first expression clearly emphasizes that we are able to compute a non-constant Boussinesq coefficient. Indeed from (2.17) we have

$$
\beta=1+\bar{\delta} \frac{\left(\delta_{1}-\delta_{2}-\bar{\delta} \delta_{1}^{2} / h\right) u_{e}^{2}}{h U^{2}}=1+\left(1-\frac{1}{H}\right) \frac{\bar{\delta} \delta_{1}}{h}+O\left(\bar{\delta}^{2}\right) .
$$

For $\bar{\delta}=0$ we recover the classical shallow water system with Boussinesq coefficient equal to 1 . As soon as viscosity effects arise, that is $\bar{\delta}>0$, we have not only the friction term on the right-hand side of (4.6) but also 
a correction of the same order one in $\bar{\delta}$ to the hydrostatic pressure. Notice that in [51] a similar correction in the flux of the shallow water system is proposed to improve the study of roll-waves.

In summary, we obtain two equivalent formulations for the extended model, both based on the following three equations, where we have used the second expression for the flux $J$ in (4.3) to write (4.6):

$$
\begin{aligned}
\partial_{t} h+\partial_{x}(h U) & =0 \\
\partial_{t}(h U)+\partial_{x}\left(h U^{2}+\bar{\delta} \delta_{1}\left(1-\frac{1}{H}-\bar{\delta} \delta_{1} / h\right) u_{e}^{2}+\frac{h^{2}}{2 \mathrm{Fr}^{2}}\right) & =-h \partial_{x} p-\bar{\delta} \tau_{b} \\
\partial_{t} u_{e}+u_{e} \partial_{x} u_{e} & =-\partial_{x} p,
\end{aligned}
$$

The difference lies now in the way to compute the displacement thickness $\delta_{1}$. The first possibility consists in adding the algebraic relation (3.8), $h U=\left(h-\bar{\delta} \delta_{1}\right) u_{e}$, which gives somewhat an equation of state. The second one makes use of the von Kármán equation that we rewrite in the following form

$$
\partial_{t}\left(\delta_{1} u_{e}\right)+\partial_{x}\left(\left(1+\frac{1}{H}\right) \delta_{1} u_{e}^{2}\right)=\bar{\tau}_{b}+u_{e} \partial_{x}\left(\delta_{1} u_{e}\right)
$$

We propose now two other models which involve only the inviscid velocity $u_{e}$, and turn out to be more tractable from both analytical and numerical viewpoints.

\subsection{Two other equivalent formulations}

The aim of this section is to propose extended shallow water (ESW) models involving an inviscid fluid with velocity $u_{e}$ and to couple them with von Kármán equation describing the evolution of the displacement thickness $\delta_{1}$. The first step towards these models consists in providing two systems where we keep equation (2.14) for mass conservation and constitutive relation (3.8) on the displacement thickness. With these relations, it can be checked that among the three following equations: inviscid momentum (4.7), viscous momentum (4.6) and von Kármán (4.8), once two equations are satisfied then so is the third one.

From this consideration, the ESW can be expressed with two equivalent formulations: the first one describes an inviscid fluid living on a viscous layer, which becomes some apparent topography; the second one represents an equivalent inviscid fluid over the whole depth $h$. It is noteworthy that the mass and momentum equations of these models can be derived as well from the Euler system with appropriate boundary conditions, as we shall see below.

Apparent topography formulation. Let us derive the first system which describes an inviscid fluid lying above the viscous layer of thickness $\bar{\delta} \delta_{1}$, see again Figure 2. First, we define the effective depth $\mathcal{H}=h-\bar{\delta} \delta_{1}$ of the inviscid fluid. The constitutive relation (3.8) allows to write the mass flux $h U=\mathcal{H} u_{e}$. Next, multiplying the inviscid fluid equation (4.7) by $\mathcal{H}$ and substituting the mass balance equation (2.14) into it, the resulting equation together with the mass balance equations and the von Kármán equation form the following coupled system

$$
\left\{\begin{array}{l}
\partial_{t} \mathcal{H}+\partial_{x}\left(\mathcal{H} u_{e}\right)+\partial_{t}\left(\bar{\delta} \delta_{1}\right)=0, \\
\partial_{t}\left(\mathcal{H} u_{e}\right)+\partial_{x}\left(\mathcal{H} u_{e}^{2}+\frac{\mathcal{H}^{2}}{2 \mathrm{Fr}^{2}}\right)+u_{e} \partial_{t}\left(\bar{\delta} \delta_{1}\right)=-\frac{\mathcal{H}}{\operatorname{Fr}^{2}}\left(f_{b}^{\prime}+\partial_{x}\left(\bar{\delta} \delta_{1}\right)\right), \\
\partial_{t}\left(\delta_{1} u_{e}\right)+\partial_{x}\left(\left(1+\frac{1}{H}\right) \delta_{1} u_{e}^{2}\right)=u_{e} \partial_{x}\left(\delta_{1} u_{e}\right)+\bar{\tau}_{b} .
\end{array}\right.
$$

One can see that the two first equations of the system represent a shallow flow, of thickness $\mathcal{H}$, over a modified topography, namely $f_{b}+\bar{\delta} \delta_{1}$. This has to be related to the so-called "apparent topography" formulation, where for numerical purposes the friction term is rewritten as the derivative of some function, see $[4,5]$. Here this derivative arises in a natural way, together with additional time derivatives in the mass and momentum equations. 
As mentioned, the apparent topography formulation can be obtained as well from integration of the Euler system over the vertical, but from the modified topography $f_{b}+\bar{\delta} \delta_{1}$ to the free surface $\eta$. The key point is that the boundary condition on the interface between the viscous layer and the inviscid fluid is a non-penetration condition

$$
\left.v\right|_{y=f_{b}+\bar{\delta} \delta_{1}}=u_{e}\left(f_{b}^{\prime}+\partial_{x}\left(\bar{\delta} \delta_{1}\right)\right)
$$

Interactive Boundary Layer formulation. This model describes an inviscid fluid on the whole water depth $h$. Multiplying (4.8) by $\bar{\delta}$ and substituting it into (4.6) leads to a model very similar to the usual shallow water one. The resulting system reads

$$
\left\{\begin{array}{l}
\partial_{t} h+\partial_{x}\left(h u_{e}-\bar{\delta} \delta_{1} u_{e}\right)=0 \\
\partial_{t}\left(h u_{e}\right)+\partial_{x}\left(h u_{e}^{2}+\frac{h^{2}}{2 \mathrm{Fr}^{2}}\right)=-\frac{h f_{b}^{\prime}}{\mathrm{Fr}^{2}}+u_{e} \partial_{x}\left(\bar{\delta} \delta_{1} u_{e}\right) \\
\partial_{t}\left(\delta_{1} u_{e}\right)+\partial_{x}\left(\left(1+\frac{1}{H}\right) \delta_{1} u_{e}^{2}\right)=u_{e} \partial_{x}\left(\delta_{1} u_{e}\right)+\bar{\tau}_{b}
\end{array}\right.
$$

An noticeable feature of this formulation is that the friction is no longer explicitly present in the momentum equation, it is replaced by an advection term on the "momentum" $\delta_{1} u_{e}$. This term actually represents the momentum exchange between these two layers. System (4.11) enjoys a more conservative structure than the previous formulation, which makes it more suitable for numerical discretization.

This formulation of the model can also be obtained by integrating the hydrostatic Euler system over the whole water depth, but the non-penetration condition (2.12) has to be replaced by a slightly modified one, called transpiration condition, which reads

$$
\left.v\right|_{y=f_{b}}=u_{e} f_{b}^{\prime}+\bar{\delta} \partial_{x}\left(\delta_{1} u_{e}\right)
$$

Notice that this transpiration condition, as it contains the displacement thickness $\delta_{1}$, is known a posteriori after performing the analysis on the boundary layer equations. So, this boundary condition helps us rather to understand how the dynamics of inviscid fluid is modified by viscous layer. This is in fact a formulation of the "Interactive Boundary Layer" (IBL) or "Viscous Inviscid Interaction" in aerodynamics, see [39]. Comparing with (2.12), one can see that the transpiration condition is a correction of order one in $\bar{\delta}$ of the non-penetration condition due to the development of viscous layer. We shall refer to (4.11) as the IBL formulation in the following.

For the sake of completeness, we briefly recall how condition (4.12) is derived. We first notice that, since $\partial_{y} u_{e}=0$, integrating incompressibility equation (2.4) of inviscid fluid over the whole water depth yields

$$
\left.v\right|_{y=f_{b}}=\left.v\right|_{y=\eta}+\left(\eta-f_{b}\right) \partial_{x} u_{e}
$$

Next, we have on the one hand

$$
\left.\bar{\delta} \bar{v}\right|_{\bar{y}=\bar{\eta}}=\left.v\right|_{y=\eta}-f_{b}^{\prime} u_{e}
$$

from the Prandtl shift (3.1). On the other hand the vertical velocity $\bar{v}$ is recovered through the incompressibility relation (3.3) in the viscous layer, since we can write $\partial_{\bar{y}} \bar{v}=\partial_{\bar{x}}\left(u_{e}-\bar{u}\right)-\partial_{\bar{x}} u_{e}$. Integrating this later equation over the depth we obtain

$$
\left.\bar{\delta} \bar{v}\right|_{\bar{y}=\bar{\eta}}=\bar{\delta} \partial_{x}\left(u_{e} \delta_{1}\right)-\left(\eta-f_{b}\right) \partial_{x} u_{e}
$$

Putting things together leads to the required transpiration boundary condition (4.12).

As for the classical shallow water model, it is straightforward to obtain an energy balance equation for (4.11) from the first two equations of system (4.11). The result reads:

$$
\partial_{t}\left[\left(h-\bar{\delta} \delta_{1}\right) \frac{u_{e}^{2}}{2}+\frac{h^{2}}{2 \mathrm{Fr}^{2}}+\frac{h f_{b}}{\mathrm{Fr}^{2}}\right]+\partial_{x}\left[u_{e}\left(h-\bar{\delta} \delta_{1}\right)\left(\frac{u_{e}^{2}}{2}+\frac{h+f_{b}}{\mathrm{Fr}^{2}}\right)\right]=-\frac{u_{e}^{2}}{2} \partial_{t}\left(\bar{\delta} \delta_{1}\right) .
$$


Since $h-\bar{\delta} \delta_{1}=\mathcal{H}$, equation (4.13) is also an energy balance equation for the apparent topography formulation.

In contrast with the classical shallow water system, the "dissipation" of energy here is driven by the dynamical behaviour in time of the displacement thickness $\delta_{1}$. If $\partial_{t}\left(\bar{\delta} \delta_{1}\right) \geq 0$, as for the Blasius-Stokes solution presented below in Section 6.2, we have actually dissipation of energy by the bottom friction, thus some stability of the solutions. If this not the case, the problem of energy dissipation is open.

\section{NumericAl ANALYSIS OF THE IBL FORMULATION}

We propose here a numerical implementation of the IBL formulation (4.11). This model was chosen because of its relative simplicity compared to the apparent topography and to the four equations models. Also, it can be related to the previous implementation in the context of rigid pipes, see [41]. In order to design a finite volume solver, we first rewrite this system in vector form as

$$
\partial_{t} W+\partial_{x} F(W)+B(W)=\tau(W),
$$

where the conservative variable $W$, the flux $F(W)$, the convective term $B(W)$ and the source term $\tau(W)$ are defined by

$$
W=\left(\begin{array}{c}
h \\
h u_{e} \\
\delta_{1} u_{e}
\end{array}\right), \quad F(W)=\left(\begin{array}{c}
h u_{e}-\bar{\delta} \delta_{1} u_{e} \\
h u_{e}^{2}+\frac{h^{2}}{2 \operatorname{Fr}^{2}} \\
\left(1+\frac{1}{H}\right) \delta_{1} u_{e}^{2}
\end{array}\right), \quad B(W)=-\left(\begin{array}{c}
0 \\
u_{e} \partial_{x}\left(\bar{\delta} \delta_{1} u_{e}\right) \\
u_{e} \partial_{x}\left(\delta_{1} u_{e}\right)
\end{array}\right), \quad \tau(W)=\left(\begin{array}{c}
0 \\
-\frac{h f_{b}^{\prime}}{\mathrm{Fr}^{2}} \\
\frac{f_{2}}{\delta_{1}} u_{e}
\end{array}\right) .
$$

The system is numerically solved by a splitting method. First we solve the so-called convective part

$$
\partial_{t} W+\partial_{x} F(W)+B(W)=0
$$

together with the topography source term $-\frac{h f_{b}^{\prime}}{\mathrm{Fr}^{2}}$, in a well-balanced way. Next, we solve the friction part

$$
\partial_{t}\left(\delta_{1} u_{e}\right)=\frac{f_{2} H}{\delta_{1}} u_{e}
$$

by a semi-implicit method.

\subsection{Eigenvalue analysis}

For numerical purpose and stability analysis, we first study the hyperbolicity of the convective part (5.1) of the model. To this end, we rewrite (5.1) in quasi-linear form $\partial_{t} W+A(W) \partial_{x} W=0$. The system is said to be hyperbolic if the convective matrix $A(W)$ is $\mathbb{R}$-diagonalizable, and strictly hyperbolic if the eigenvalues are distinct. Estimating these eigenvalues is also important to design an explicit finite volume scheme for the system. As eigenvalues are invariant by changing variables [25], it is therefore more convenient to make the variable change $W \mapsto Y(W)=\left(h, u_{e}, \delta_{1} u_{e}\right)^{t}$ and study eigenvalues of the corresponding convective matrix

$$
\tilde{A}(Y)=\left(\begin{array}{ccc}
u_{e} & h & -\bar{\delta} \\
\operatorname{Fr}^{-2} & u_{e} & 0 \\
0 & a & b-u_{e}
\end{array}\right)
$$

where we have denoted the partial derivatives

$$
a=\frac{\partial}{\partial u_{e}}\left(\left(1+\frac{1}{H}\right) \delta_{1} u_{e}^{2}\right), \quad b=\frac{\partial}{\partial\left(\delta_{1} u_{e}\right)}\left(\left(1+\frac{1}{H}\right) \delta_{1} u_{e}^{2}\right) .
$$

Their explicit expressions can be computed as well once a closure formula for the shape factor is provided, since the shape factor $H$ is a function of $\Lambda_{1}$, so $H$ depends only on $u_{e}$ and $\delta_{1} u_{e}$. 


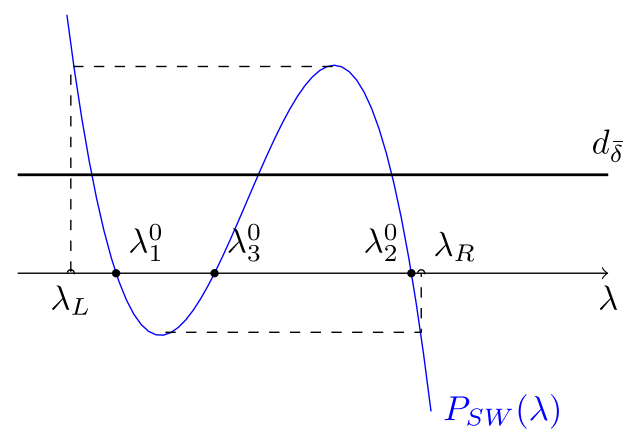

FIGURE 5. Roots of characteristic polynomial of the coupled system.

The characteristic polynomial of $\tilde{A}(Y)$ reads

$$
\begin{aligned}
P(\lambda)=\operatorname{det}(\tilde{A}-\lambda \mathrm{Id}) & =-\left[\left(b-u_{e}-\lambda\right)\left(\left(u_{e}-\lambda\right)^{2}-\mathrm{Fr}^{-2} h\right)-\bar{\delta} \mathrm{Fr}^{-2} a\right] \\
& =-P_{\mathrm{SW}}(\lambda)+d_{\bar{\delta}},
\end{aligned}
$$

where

$$
P_{\mathrm{SW}}(\lambda)=\left(b-u_{e}-\lambda\right)\left(\left(u_{e}-\lambda\right)^{2}-\mathrm{Fr}^{-2} h\right), \quad d_{\bar{\delta}}=\bar{\delta} \mathrm{Fr}^{-2} a .
$$

The polynomial $P_{\mathrm{SW}}$ has always 3 roots, denoted $\lambda_{1,2,3}^{0}$, which represent the wave speeds of the system with no coupling between shallow water equations and the von Kármán equation. Indeed, the first two roots $\lambda_{1,2}^{0}$ express the propagation velocities of inviscid fluid while the last one $\lambda_{3}^{0}$ approximates that of the viscous layer:

$$
\lambda_{1,2}^{0}=u_{e} \pm \frac{\sqrt{h}}{\mathrm{Fr}}, \quad \lambda_{3}^{0}=b-u_{e} .
$$

From this it follows that, for $\bar{\delta}$ small enough, the characteristic polynomial admits 3 real eigenvalues and the system turns out to be hyperbolic. More precisely, denoting $\lambda_{-}<\lambda_{+}$the roots of $P_{\mathrm{SW}}^{\prime}(\lambda)$, the system is hyperbolic when $d_{\bar{\delta}}$ lies between $P_{\mathrm{SW}}\left(\lambda_{ \pm}\right)$, see Figure 5 , that is

$$
P_{\mathrm{SW}}\left(\lambda_{-}\right)<\frac{\bar{\delta} a}{\operatorname{Fr}^{2}}<P_{\mathrm{SW}}\left(\lambda_{+}\right) .
$$

It is straightforward to verify that for the case of closure (3.17) based on Falkner-Skan solutions, the third wave speed reads

$$
\lambda_{3}^{0}=\frac{u_{e}}{H}\left(1+0.74 \Lambda_{1}\right), \quad \text { for } \Lambda_{1}<0.6,
$$

and in particular when $\Lambda_{1}=0$, i.e. the Blasius solution, the viscous layer propagates downstream at velocity $\lambda_{3}^{0}=u_{e} / H \simeq 0.39 u_{e}$ (see Fig. 6-left).

Even within the hyperbolic regime, the eigenstructure is given only implicitly, due to the form of nonlinear coupling between the inviscid fluid and viscous layer. We propose hereafter a HLL type scheme [29] taking advantage that the numerical flux arises directly from the governing equations (5.1) and only an estimation of lowest and the largest wave speeds $\lambda_{L, R}$ is required. Such an estimate can be obtained by using accurate Nickalls's bounds [47], which finally lead to

$$
\lambda_{L, R}(W)=\frac{1}{3}\left(u_{e}+b \mp 2 \sqrt{\left(2 u_{e}-b\right)^{2}+\frac{3 h}{\mathrm{Fr}^{2}}}\right), \quad b=u_{e}\left(1+\frac{1+0.74 \Lambda_{1}}{H}\right),
$$

in which we have used (5.4) provided from the Falkner-Skan closure. 

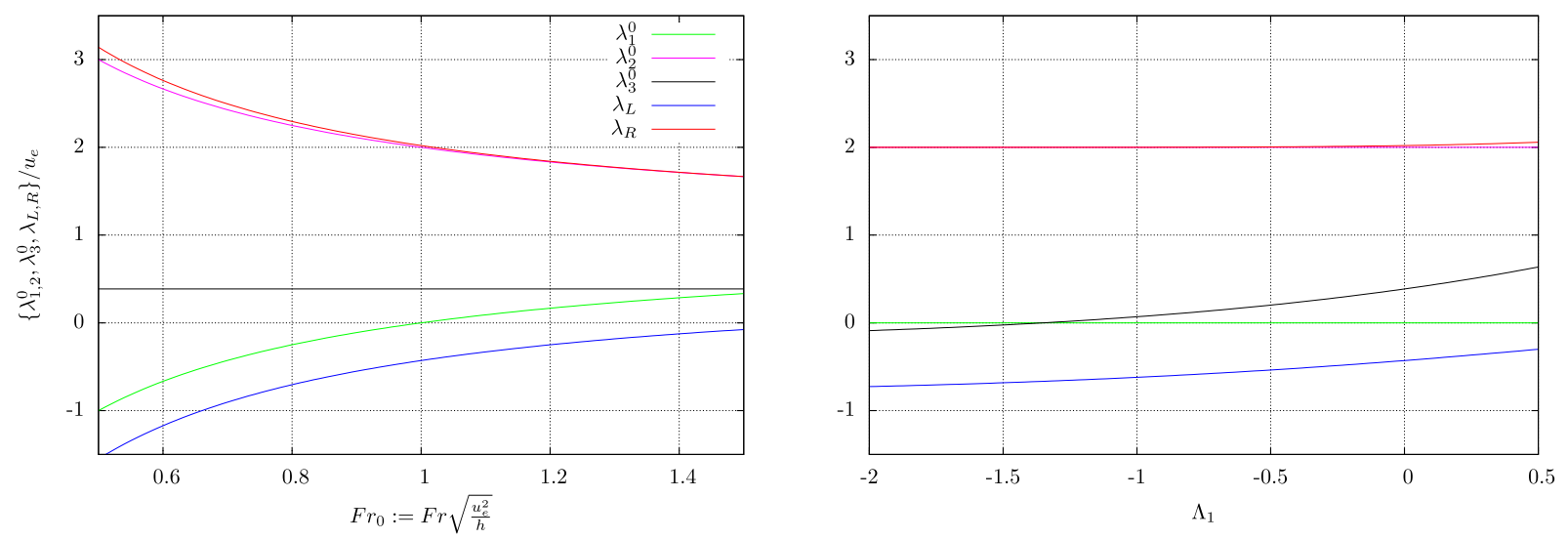

Figure 6. Comparison of wave speeds $\lambda_{1,2,3}^{0}$ and the estimations $\lambda_{L, R}$. Left panel: Blasius case $\left(\Lambda_{1}=0\right)$, wave speeds as a function of the Froude nimber; Right panel: critical flow $\left(\operatorname{Fr}_{0}=1\right)$, wave speeds as a function of $\Lambda_{1}$ (same color code on both graphs, color online).

On Figure 6 we compare the shallow water wave speeds $\lambda_{1,2}^{0}$, the viscous layer one $\lambda_{3}^{0}$ and the bounds $\lambda_{L, R}$ given by (5.5). Rescaling these velocities by $u_{e}$, the result can be considered as functions of the local Froude number $\operatorname{Fr}_{0}=\operatorname{Fr} \sqrt{u_{e}^{2} / h}$ and the pressure parameter $\Lambda_{1}$. The left figure shows the dependence on $\operatorname{Fr}_{0}$ in the case of Blasius solution, i.e. $\Lambda_{1}=0$, both for subcritical and supercritial regimes. We find that the estimation $\lambda_{R}$ for the largest wave speed is very accurate. On the right figure, we display the dependence on $\Lambda_{1}$ for a fixed value of $\mathrm{Fr}_{0}$, e.g. by considering the case of critical flow so $\lambda_{1}^{0}=0$. As we can see, the velocity $\lambda_{3}^{0}$ can be negative the wave associated to the viscous layer propagates upstream - for large reverse flow.

\subsection{A Godunov-type finite volume scheme}

Let us recall some basic notations of finite volume discretization. We introduce a space step $\Delta x$ and a time step $\Delta t$, both assumed to be constant for simplicity. The computational domain is discretized by a sequence of points $x_{j+1 / 2}=j \Delta x$ for $j \in \mathbb{Z}$. We define $W_{j}^{0}$ a piecewise constant approximation of initial condition on each control volume $\left.C_{j}=\right] x_{j-1 / 2}, x_{j+1 / 2}[$.

Convection step. Assume that the solution $W_{j}^{n}$ at time $t^{n}$ is known. The first-order three-points finite volume scheme writes

$$
W_{j}^{n+1 / 2}=W_{j}^{n}-\frac{\Delta t}{\Delta x}\left(F_{j+1 / 2}^{L}-F_{j-1 / 2}^{R}\right),
$$

where the left- and right- numerical fluxes $F_{j+1 / 2}^{L, R}=F^{L, R}\left(W_{j}^{n}, W_{j+1}^{n}\right)$ are to be determined. Godunov-type schemes allow to compute these fluxes through an approximate solution $W_{\Delta}(t, x)$ of the exact solution $W_{\mathcal{R}}(t, x)$ of the local Riemann problem at each interface $x_{j+1 / 2}$.

Given initial data $\left(W_{L}, W_{R}\right)$ of local Riemann problem, we adopt a simple Riemann solver $W_{\Delta}(t, x)$ consisting of three discontinuity waves propagating with velocities $\lambda_{L} \leq 0, \lambda_{0}=0, \lambda_{R} \geq 0$, where $\lambda_{L, R}$ are given by formula (5.5), and two intermediate states $W_{L}^{*}$ and $W_{R}^{*}$ in the star region, see Figure 7 . The zero velocity $\lambda_{0}$ corresponds to the stationary contact discontinuity associated with the topography. The third eigenvalue $\lambda_{3}$ was not used here because the analytical expression of the Riemann invariant is unknown.

In order to avoid interaction between adjacent approximate Riemann problems, the time step $\Delta t$ must satisfiy the CFL type condition

$$
\Delta t \leq \frac{\Delta x}{2 \max \left|\lambda_{L, R}\right|}
$$




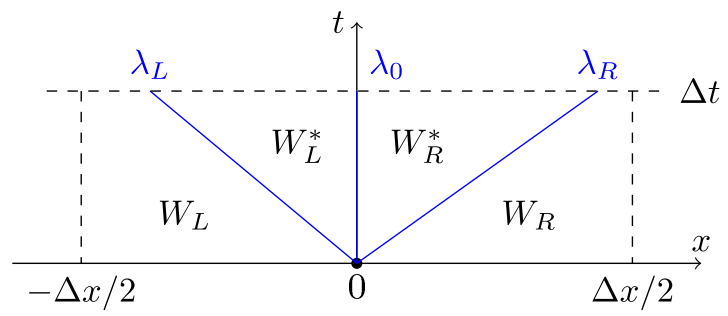

FiguRE 7. A three-waves approximate Riemann problem.

Then the numerical fluxes are given by

$$
\begin{aligned}
& F^{L}\left(W_{L}, W_{R}\right)=F\left(W_{L}\right)+\lambda_{L}\left(W_{L}^{*}-W_{L}\right), \\
& F^{R}\left(W_{L}, W_{R}\right)=F\left(W_{R}\right)-\lambda_{R}\left(W_{R}-W_{R}^{*}\right) .
\end{aligned}
$$

Therefore, designing such a scheme consists in determinating the intermediate states $W_{L, R}^{*}$ in the star region. Notice that, if the intermediate depths are nonnegative, the positivity of updated solution is preserved.

According to Harten et al. [29] the approximate solver $W_{\Delta}(t, x)$ must be consistent with the exact solution $W_{\mathcal{R}}(t, x)$ in the sense that

$$
\int_{-\frac{\Delta x}{2}}^{\frac{\Delta x}{2}} W_{\mathcal{R}}(\Delta t, x) \mathrm{d} x=\int_{-\frac{\Delta x}{2}}^{\frac{\Delta x}{2}} W_{\Delta}(\Delta t, x) \mathrm{d} x .
$$

Applying to the conservation law (5.1), this integral consistency condition provides that the intermediate states satisfy the following relations

$$
\begin{aligned}
\lambda_{R} h_{R}^{*}-\lambda_{L} h_{L}^{*} & =\lambda_{R} h_{R}-\lambda_{L} h_{L}-\left[\left[h u_{e}-\bar{\delta} \delta_{1} u_{e}\right]\right] \\
\lambda_{R}\left(h u_{e}\right)_{R}^{*}-\lambda_{L}\left(h u_{e}\right)_{L}^{*} & =\lambda_{R}\left(h u_{e}\right)_{R}-\lambda_{L}\left(h u_{e}\right)_{L}-\left[\left[h u_{e}^{2}+\frac{h^{2}}{2 \operatorname{Fr}^{2}}\right]\right]-\Delta x\left\{\frac{h f_{b}^{\prime}}{\operatorname{Fr}^{2}}\right\}+\bar{\delta} \Delta x\left\{u_{e} \partial_{x}\left(\delta_{1} u_{e}\right)\right\}, \\
\lambda_{R}\left(\delta_{1} u_{e}\right)_{R}^{*}-\lambda_{L}\left(\delta_{1} u_{e}\right)_{L}^{*} & =\lambda_{R}\left(\delta_{1} u_{e}\right)_{R}-\lambda_{L}\left(\delta_{1} u_{e}\right)_{L}-\left[\left[\left(1+\frac{1}{H}\right) \delta_{1} u_{e}^{2}\right]\right]+\Delta x\left\{u_{e} \partial_{x}\left(\delta_{1} u_{e}\right)\right\}
\end{aligned}
$$

provided the space-time averaging of the non-conservative source terms, and $\llbracket \bullet \rrbracket=(\bullet)_{R}-(\bullet)_{L}$ standings for the usual jump operator.

Recall that that the non-conservative products arising in the source terms may not make sense as distributions. Dal Maso et al. [13] proposed an interpretation of these products as Borel measures based on the choice of paths $\Phi\left(s ; W_{L}, W_{R}, f_{b L}, f_{b R}\right)=\left(\Phi_{h}, \Phi_{h u_{e}}, \Phi_{\delta_{1} u_{e}}, \Phi_{f_{b}}\right), s \in[0,1]$, in the phase space. This actually consists in specifying appropriate parameterizations of $W$ and $f_{b}$ through the discontinuity. Once the choice is made, space-time averaging of the source terms reads

$$
\begin{gathered}
\Delta x\left\{\frac{h f_{b}^{\prime}}{\operatorname{Fr}^{2}}\right\}=\frac{1}{\Delta t} \int_{-\frac{\Delta x}{2}}^{\frac{\Delta x}{2}} \int_{0}^{\Delta t} \frac{h f_{b}^{\prime}}{\operatorname{Fr}^{2}} \mathrm{~d} t \mathrm{~d} x=\int_{0}^{1} \frac{\Phi_{h}}{\operatorname{Fr}^{2}} \frac{\partial \Phi_{f_{b}}}{\partial s} \mathrm{~d} s \\
\Delta x\left\{u_{e} \partial_{x}\left(\delta_{1} u_{e}\right)\right\}=\frac{1}{\Delta t} \int_{-\frac{\Delta x}{2}}^{\frac{\Delta x}{2}} \int_{0}^{\Delta t} u_{e} \partial_{x}\left(\delta_{1} u_{e}\right) \mathrm{d} t \mathrm{~d} x=\int_{0}^{1} \frac{\Phi_{h u_{e}}}{\Phi_{h}} \frac{\partial \Phi_{\delta_{1} u_{e}}}{\partial s} \mathrm{~d} s .
\end{gathered}
$$

It is worth mentioning that the choice of paths is important and has to be based on the physical background since it determines the speed of propagation of shocks. A convenient way is to construct these parameterizations from the integral curves of the characteristic fields because the resulting Godunov-type scheme is well-balanced [44] and, in particular, is able to preserve moving steady states (i.e. with non-zero velocity). In constrast with 
the classical shallow water model, the characteristic fields of extended models are still not known analytically due to the nonlinearity of the von Kármán equation, so that building desirable paths for such a scheme is more difficult. Moreover, the proposed Falkner-Skan closure (3.17), as it involves the derivative $\partial_{x} u_{e}$ when computing $\Lambda_{1}$, is not relevant for the case of shock wave.

Therefore, since investigating the sensitivity on the choice of paths and building an advanced numerical scheme are not the first priorities of the present study, we limit ourselves in this work to use the simplest and most classical path family, namely Vol'pert's one [59]. This choice leads to the following approximations

$$
\Delta x\left\{\frac{h f_{b}^{\prime}}{\mathrm{Fr}^{2}}\right\}=\frac{h_{L}+h_{R}}{2 \mathrm{Fr}^{2}} \llbracket f_{b} \rrbracket, \quad \Delta x\left\{u_{e} \partial_{x}\left(\delta_{1} u_{e}\right)\right\}=\frac{\left(h u_{e}\right)_{L}+\left(h u_{e}\right)_{R}}{h_{L}+h_{R}} \llbracket \delta_{1} u_{e} \rrbracket,
$$

which preserve at least the so-called lake-at-rest equilibrium state. This somewhat naive choice is sufficient for our illustrative purpose in the following, being aware that further research on numerical method is needed.

Consistency conditions (5.9)-(5.11) have to be complemented by three additional relations in order to solve completly the intermediate states. The Riemann invariants associated to the stationary contact wave can be used to provide these missing relations

$$
\begin{aligned}
\left(h u_{e}\right)_{L}^{*}-\bar{\delta}\left(\delta_{1} u_{e}\right)_{L}^{*} & =\left(h u_{e}\right)_{R}^{*}-\bar{\delta}\left(\delta_{1} u_{e}\right)_{R}^{*} \\
\left(\frac{\left(u_{e}\right)_{R}^{* 2}}{2}+\frac{h_{R}^{*}}{\mathrm{Fr}^{2}}\right)-\left(\frac{\left(u_{e}\right)_{L}^{* 2}}{2}+\frac{h_{L}^{*}}{\mathrm{Fr}^{2}}\right) & =-\frac{\llbracket f_{b} \rrbracket}{\mathrm{Fr}^{2}} \\
\left(\delta_{1} u_{e}\right)_{L}^{*} & =\left(\delta_{1} u_{e}\right)_{R}^{*}
\end{aligned}
$$

Note that the first two equations are the analytical Riemann invariants of the system applied for intermediate states while the third one related to $\delta_{1} u_{e}$ is just an approximation. In fact, the last analytical Riemann invariant is not known explicitly due to the form of nonlinear coupling between the inviscid fluid and viscous layer. From the numerical point of view, this consists in approximating $\left(\delta_{1} u_{e}\right)_{L, R}^{*}$ in the star region by only one averaged value $\left(\delta_{1} u_{e}\right)^{*}$, as in the classical HLL scheme [29]. Moreover, this choice leads to $\left(h u_{e}\right)_{L}^{*}=\left(h u_{e}\right)_{R}^{*}=q^{*}$ and, together with (5.14), we find again the well-known Bernoulli relations of shallow water equations

$$
\frac{q^{* 2}}{2}\left(\frac{1}{h_{R}^{* 2}}-\frac{1}{h_{L}^{* 2}}\right)+\frac{h_{R}^{*}-h_{L}^{*}}{\mathrm{Fr}^{2}}=-\frac{\llbracket f_{b} \rrbracket}{\mathrm{Fr}^{2}} .
$$

Plugging (5.13), (5.15) into (5.10) and (5.11) allows us to solve the discharges $q^{*}$ and $\left(\delta_{1} u_{e}\right)^{*}$ in the star region. Next, non-negative intermediate depths $h_{L, R}^{*}$ are obtained from (5.9) and (5.16). Similarly to the case of classical shallow water model, it can be shown that the present scheme is accurate and well-balanced. We refer to [26] for more technical details and discussions.

Friction step. Once the convection step (5.6) is done, the next step is to take into account the friction source term of the von Kármán equation to modify the displacement thickness. This consists in solving equation (5.2) with initial data $W^{n+1 / 2}$ to get the solution $W^{n+1}$ at the next time step. To this end, we use a simple semi-implicit scheme which writes

$$
\begin{gathered}
h^{n+1}=h^{n+1 / 2}, \quad u_{e}^{n+1}=u_{e}^{n+1 / 2}, \\
\frac{\left(\delta_{1} u_{e}\right)^{n+1}-\left(\delta_{1} u_{e}\right)^{n+1 / 2}}{\Delta t}=\frac{\left(f_{2} H\right)^{n}}{\delta_{1}^{n+1}} u_{e}^{n+1 / 2} .
\end{gathered}
$$

This discretization leads to a second-order equation for $\delta_{1}^{n+1}$. Under condition $\left(\delta_{1}^{n+1 / 2}\right)^{2}+4\left(f_{2} H\right)^{n} \Delta t \geq 0$, which implies an additional restriction on $\Delta t$ only in the case of reverse flow

$$
\Delta t \leq-\frac{\left(\delta_{1}^{n+1 / 2}\right)^{2}}{4\left(f_{2} H\right)^{n}} \quad \text { if } \quad f_{2}^{n}<0,
$$


this equation has two solutions, from which only one is physically admissible

$$
\delta_{1}^{n+1}=\frac{1}{2}\left(\delta_{1}^{n+1 / 2}+\sqrt{\left(\delta_{1}^{n+1 / 2}\right)^{2}+4\left(f_{2} H\right)^{n} \Delta t}\right) .
$$

In practice when using the Falkner-Skan closure, condition (5.17) is not restrictive, the time step is rather controlled by the CFL condition (5.7) of the convection step.

\subsection{Multi layer formulation}

We conclude this section by a short presentation of the so-called multi-layer Shallow Water (MLSW) model proposed by [2], which we shall use as a reference for comparison in the next section. The multi-layer model considers a superposition of shallow water systems each one interacting with its neighbours. As proposed by the authors, the friction term $\tau_{\alpha+1 / 2}$ between layers $\alpha$ and $\alpha+1$ is discretized in an implicit way by

$$
\tau_{\alpha+1 / 2}=\bar{\delta}^{2} \frac{2\left(u_{\alpha+1}-u_{\alpha}\right)}{h_{\alpha+1}+h_{\alpha}} \quad \text { for } \quad 0<\alpha<N, \quad \tau_{b}=\tau_{1 / 2}=\bar{\delta}^{2} \frac{2 u_{1}}{h_{1}}, \quad \tau_{N+1 / 2}=0,
$$

in which $h_{\alpha}=\ell_{\alpha} h$ denotes the layer thickness, $\ell_{\alpha}>0$ being a given constant, $\sum_{\alpha=1}^{N} \ell_{\alpha}=1$, and $u_{\alpha}$ the averaged velocity in layer $\alpha$.

Using this model imposes some constraints on the vertical discretization: it requires a very thin layers near the bottom in order to accurately compute the friction while the velocity of two adjacent layers (in the viscous region) must not be too different in order to preserve the hyperbolicity of the model. This later condition implies that relative thickness $\ell_{\alpha}$ of the layers can be varied but only gradually. For the present study, a discretization such as $\ell_{\alpha}=z_{\alpha}-z_{\alpha-1}$ with

$$
z_{\alpha}=\frac{\mathrm{e}^{10 \alpha / N}-1}{\mathrm{e}^{10}-1} \text { for } 0 \leq \alpha \leq N=100
$$

seems to give satisfactory results. Nevertheless the simulation with MLSW model is computationally expensive.

We interpret this MLSW system as a numerical scheme to solve the RNSP equations (3.4)-(3.6), the layers being the numerical discretization along the vertical direction (see [24] for a similar point of view in elastic tubes). In our approach, at the RNSP level, the two superposed layers have different physical properties (viscous/ inviscid fluid), and analyzed through asymptotic rescalings of the equations. At the integrated level, the closest formulation is the apparent topography, we have again two superposed layers of different physical nature, and their relative thickness, $\bar{\delta} \delta_{1} / h$, is not fixed but evolves in time, in contrast with the multi-layer model.

\section{Numerical ILLUSTRATIONS}

The aim of this last part is to give a few illustrations of the behaviour of the ESW system (4.11). We are aware that more accurate analysis is mandatory, in both the numerical approach (in particular numerical boundary conditions) and the qualitative and quantitative behaviour of the model. First we give a convergence study based on a steady-state solution. A second step is devoted to a comparison between the ESW solutions and those of the classical viscous shallow water system. Finally we compute the solutions over a small bump, in order to evidence the above mentioned phase-lag of the friction term, and the behaviour of the model with respect to various parameters.

In all test cases, we considered a very thin viscous layer by setting $\bar{\delta}=10^{-3}$. This corresponds to $\operatorname{Re}_{h}=10^{6}$ a typical value for a river, where the Reynolds number is large. The reference Froude number Fr is set to unity meaning that the longitudinal velocity was scaled by the reference celerity $\sqrt{g h_{0}}$. The computation domain was $[0, L]$; space step $\Delta x$ was specified in each test case while time step $\Delta t$ was always computed from CFL condition (5.7) to optimize computation time. Initial conditions was $h(0, x)=h_{0}, u_{e}(0, x)=1, \delta_{1}(0, x)=0 \forall x \in[0, L]$. We set $h_{0}=2$ for subcritical test cases, so $\mathrm{Fr}_{0} \simeq 0.7$, while we used $h_{0}=0.5$, i.e. $\mathrm{Fr}_{0} \simeq 1.4$, for supercritical cases. On the boundary conditions, we considered at $x=L$ a free outflow boundary. At $x=0$ for both sub- 
and supercritical flows we imposed a constant velocity $u_{e}=1$ with flat profile, so that $\delta_{1}=0$. For supercritical flows, a constant water depth $h=h_{0}$ was imposed as well. In the case of subcritial flows, $h$ was computed using the Riemann invariant of classical shallow water model. This approach is only an approximation for ESW, more in-depth study on numerical boundary condition is of course needed.

\subsection{Convergence study on a Blasius-like steady solution}

In this section we investigate an approximate stationary solution of the ESW model which is an order one perturbation of a basic stationary solution to the ESW system, namely $\left(h^{0}, u_{e}^{0}, \delta_{1}^{0}\right)$, where $h^{0}$ and $u_{e}^{0}$ are constant solutions of the frictionless shallow water system, and $\delta_{1}^{0}$ is the classical Blasius profile for the von Kármán equation on a flat plate. We look for a solution to the ESW system at first order in $\bar{\delta}$

$$
h=h^{0}+\bar{\delta} h^{1}, \quad u_{e}=u_{e}^{0}+\bar{\delta} u_{e}^{1}, \quad \delta_{1}=\delta_{1}^{0}+\bar{\delta} \delta_{1}^{1} .
$$

A very interesting feature of this solution is that the order one terms are not necessarily stationary. This gives an explicit illustration of the actual interaction between the viscous layer and the perfect fluid.

Plugging the expansion (6.1) in (4.11), we recover through the first two equations a standard inviscid shallow water model, for which a basic stationary solution consists in constant $h^{0}$ and $u_{e}^{0}$. Now we turn to the stationary von Kármán equation

$$
u_{e} \delta_{1} \partial_{x} u_{e}+\partial_{x}\left(\frac{u_{e}^{2} \delta_{1}}{H}\right)=\frac{f_{2} H}{\delta_{1}} u_{e} .
$$

In this equation, $H$ and $f_{2}$ depend on $\Lambda_{1}=\delta_{1}^{2} \partial_{x} u_{e}=\bar{\delta} \delta_{1}^{0} \partial_{x} u_{e}^{1}+O\left(\bar{\delta}^{2}\right)$. Therefore at zeroth order $H$ and $f_{2}$ are constant, so that we indeed recover the classical Blasius solution

$$
\delta_{1}^{0}=\sqrt{\frac{2 f_{2} H^{2}}{u_{e}^{0}} x}=1.718 \sqrt{x}, \quad \bar{\tau}_{b}^{0}=\frac{f_{2} H}{\delta_{1}^{0}}=\frac{0.332}{\sqrt{x}} .
$$

We use the solution $\left(h^{0}, u_{e}^{0}, \delta_{1}^{0}\right)$ as the basic solution in the expansion (6.1), and turn now to order one terms. Once again, straightforward computations lead to uncoupling the first two equations, yealding the following linearized shallow water model:

$$
\partial_{t}\left(\begin{array}{c}
h^{1} \\
u_{e}^{1}
\end{array}\right)+\left(\begin{array}{cc}
u_{e}^{0} & h^{0} \\
\frac{1}{\mathrm{Fr}^{2}} & u_{e}^{0}
\end{array}\right) \partial_{x}\left(\begin{array}{c}
h^{1} \\
u_{e}^{1}
\end{array}\right)=\left(\begin{array}{c}
\partial_{x}\left(\delta_{1}^{0} u_{e}^{0}\right) \\
0
\end{array}\right)
$$

Notice that this system has a stationary solution, given by

$$
h=h^{0}+\bar{\delta} \frac{\mathrm{Fr}_{0}^{2}}{\operatorname{Fr}_{0}^{2}-1} \delta_{1}^{0}, \quad u_{e}=u_{e}^{0}+\bar{\delta} \frac{1}{1-\mathrm{Fr}_{0}^{2}} \delta_{1}^{0}
$$

in which we have defined the local Froude number $\operatorname{Fr}_{0}=\operatorname{Fr} u_{e}^{0} / \sqrt{h^{0}}$.

We consider the computation domain $x \in[0,0.1]$. On Figure 8-left we plot the Blasius solution in which the displacement thickness $\delta_{1}^{0}$ increases in function of $\sqrt{x}$ while the friction decreases from infinity according to (6.2). On Figure 8-right we display the results of a mesh convergence study on the gap between $\delta_{1}$ and its zeroth order approximation $\delta_{1}^{0}$. The convergence study was performed in both sub- and supercritical regimes.

First we remark that when the mesh size $\Delta x$ is small enough, say $\Delta x \leq 0.1 \times 10^{-3}$, numerical results reach the model error. Indeed, we obtained at this spatial resolution that

$$
\int_{0}^{0.1}\left|\delta_{1}-\delta_{1}^{0}\right| \mathrm{d} x \simeq 0.1 \bar{\delta} \quad \text { and so } \quad \delta_{1}-\delta_{1}^{0}=O(\bar{\delta}) .
$$

Next, we notice that the supercritical case converges faster than the subcritical one. This could be explained by the fact that, on the one hand, numerical treatment of the left boundary condition is more accurate in the supercritical case as we have noticed before; on the other hand, it is well known that the HLL-type numerical flux (5.8) is also more accurate in that case. 

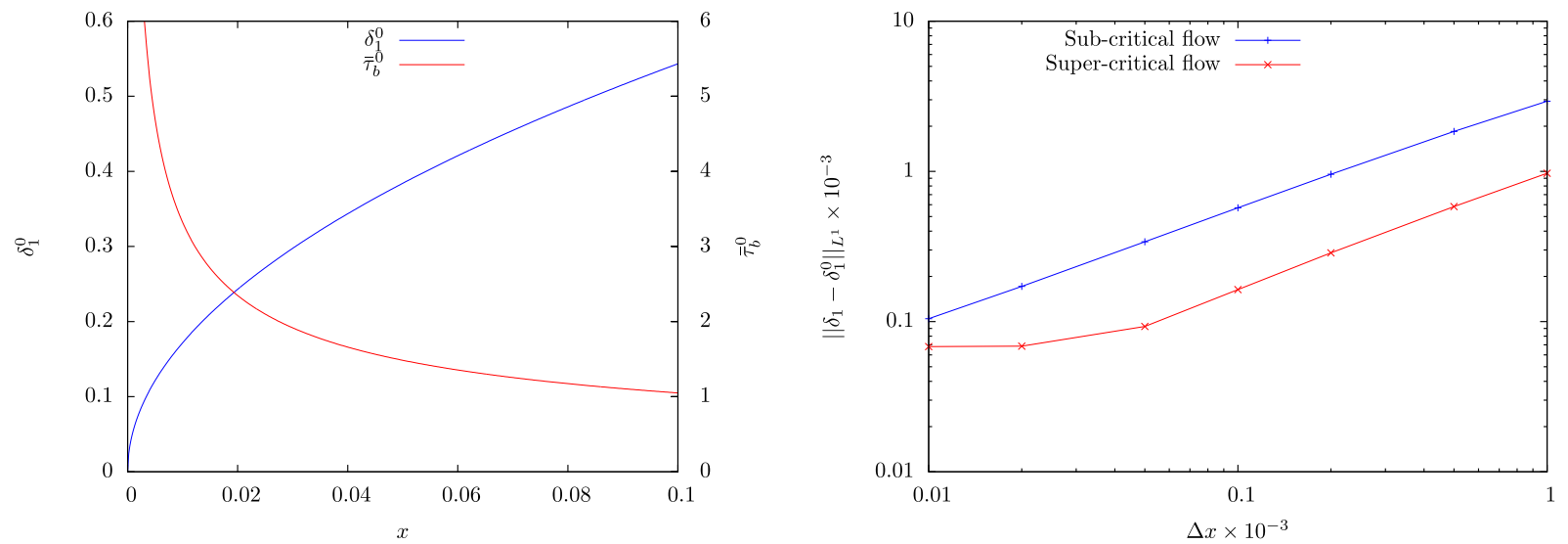

Figure 8. Left panel: Blasius solution for sake of illustration, displacement thickness $\delta_{1}^{0}=$ $1.718 \sqrt{x}$ and shear $\bar{\tau}_{b}^{0}=0.332 / \sqrt{x}$. Right panel: error $\int_{0}^{0.1}\left|\delta_{1}-\delta_{1}^{0}\right| \mathrm{d} x$ as the function of the mesh size $\Delta x$.

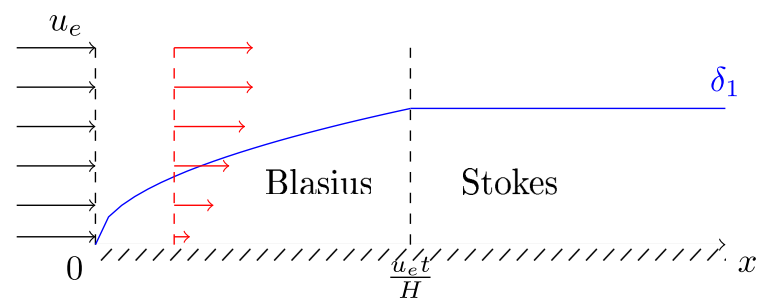

FIGURE 9. Impulsively started flow over a flat bed (Stewartson's 1951-1973 problem): transition between unsteady Stokes Rayleigh problem and steady Blasius problem.

\subsection{Impulsively started flow over a flat bed}

We turn now to a configuration introduced by Stewartson in $[54,55]$ as a simple test-case to study unsteady boundary layer solutions. It consists in a semi-infinite flow impulsively started from rest at $t=0$ with constant velocity $u_{e}$, see Figure 9 . The fluid is injected continuously at $x=0$ with a constant velocity, the flow must satisfy the no slip boundary condition for $x>0$. An accurate resolution with $\Delta x=0.1 \times 10^{-3}$ was used in order to properly capture this unsteady solution.

The solution exhibits different behaviours depending on two asymptotic regimes: for large $t$ (or small $x$ ) we recover the Blasius solution (6.2); conversely, for large $x$ (or small $t$ ), convective terms in the momentum equation (3.4) are negligible so the Prandtl system reduces to Stokes' first problem (also called Rayleigh problem by Stewartson). The solution for the velocity profile can be expressed using the erf error function:

$$
\begin{aligned}
& \frac{\bar{u}}{u_{e}}=\operatorname{erf}\left(\frac{\bar{y}}{2 \sqrt{t}}\right), \quad \delta_{1}=2 \sqrt{\frac{t}{\pi}}, \quad \bar{\tau}_{b}=\frac{1}{\sqrt{\pi t}} \\
& H=1+\sqrt{2} \simeq 2.414, \quad f_{2}=\frac{2}{\pi(1+\sqrt{2})} \simeq 0.264 .
\end{aligned}
$$

Transition between these two solutions occurs for $x=O(t)$. 

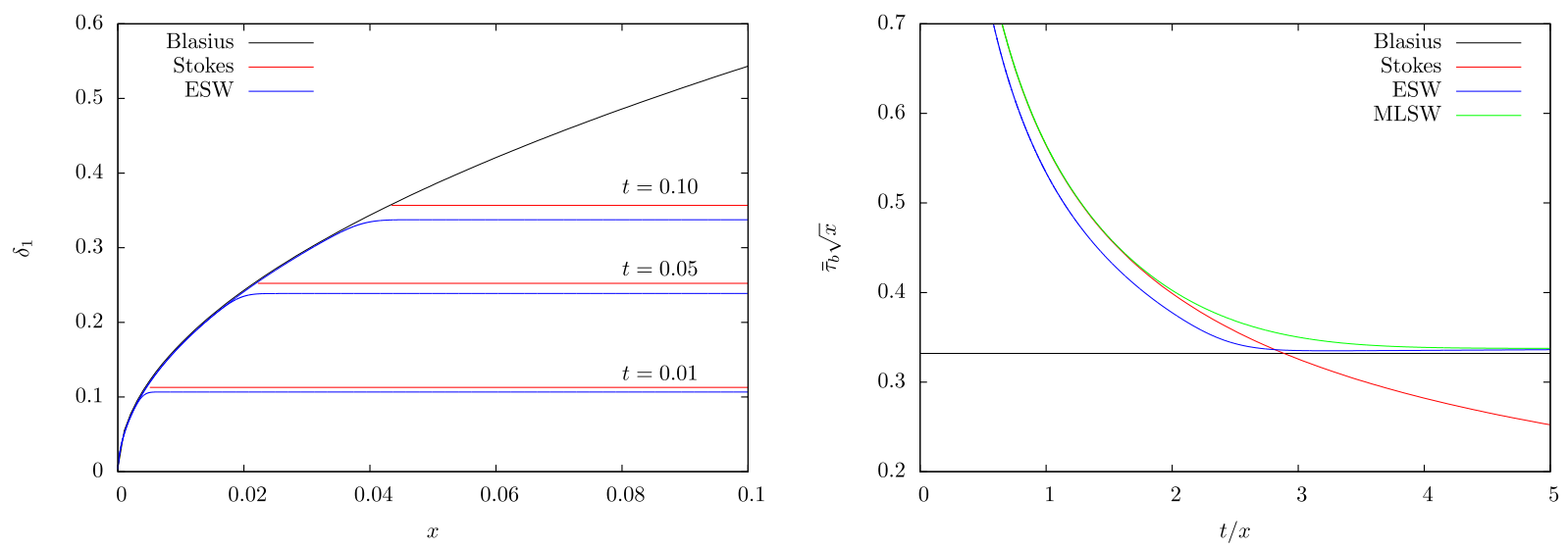

FiguRE 10. From Stokes to Blasius solution. Left panel: development of displacement thickness $\delta_{1}$ function of $x$ at different times; Right panel: comparaison of ESW and multilayer shallow water model (MLSW) for $\bar{\tau}_{b} \sqrt{x}$ in function of $t / x$ : for small $t / x$, the solution is that of Stokes $\bar{\tau}_{b} \sqrt{x}=\sqrt{x / \pi t}$; at large $t / x$ the curves collapse on the Blasius solution $\bar{\tau}_{b} \sqrt{x}=0.332$.

Stewartson noticed that an approximate integral form can be used to solve this problem. Assuming a fixed profile, e.g. Blasius (constant) value of $H$ and $f_{2}$, the von Kármán equation can be rewritten in the form

$$
\partial_{t}\left(\delta_{1}^{2}\right)+\frac{u_{e}}{H} \partial_{x}\left(\delta_{1}^{2}\right)=2 f_{2} H
$$

and it has to be complemented with the following initial and boundary value conditions:

$$
\delta_{1}(0, x)=\delta_{1}(t, 0)=0, \quad x, t \in \mathbb{R}^{+} .
$$

The solution is readily obtained by the method of characteristics:

$$
\delta_{1}=\left\{\begin{array}{l}
\sqrt{\frac{2 f_{2} H^{2} x}{u_{e}}} \text { if } x \leq \frac{u_{e} t}{H}, \\
\sqrt{2 f_{2} H t} \text { otherwise, }
\end{array} \quad \text { and } \quad \bar{\tau}_{b}=\left\{\begin{array}{l}
\sqrt{\frac{f_{2} u_{e}^{3}}{2 x}} \text { if } x \leq \frac{u_{e} t}{H}, \\
\sqrt{\frac{f_{2} H u_{e}^{2}}{2 t}} \text { otherwise }
\end{array}\right.\right.
$$

Transition zone is found at the characteristic line $x=\frac{u_{e} t}{H}$, i.e. viscous layer informations propagate at velocity $u_{e} / H$ as we have seen in equation (5.4). It is worth noticing that this solution with constant $u_{e}$ creates an unbounded viscous layer, without regards of the limitation of the water depth. Hence it is clearly physically not valid for large $x$ or $t$.

On Figure 10, we plot on the left side the evolution of the displacement $\delta_{1}$ along $x$ at different times. The two-regimes behaviour of the unsteady solution (6.5) is qualitatively well recovered: $\delta_{1}$ is constant in $x$ far from the entrance and increases in time; it reaches the Blasius solution which is steady. But, quantitatively, we observe a gap between the solution of ESW and the Stokes one, which increases in time. This is due to the choice of a fixed Blasius profile in the viscous layer. Regarding equations (6.2) and (6.4), the Blasius and Stokes profile have not exactly the same shape and this leads to different values of $H$ and $f_{2}$. More precisely, in the Stokes region the model predicts $\delta_{1}=1.067 \sqrt{t}$ while the exact solution is $\delta_{1}=1.128 \sqrt{t}$.

To illustrate the transition in profiles from Stokes to Blasius, we propose here to compare the numerical results given by the ESW model with those of the multi-layer model (MLSW) described in Section 5.3. On Figure 10, we plot on the right side the difference between the numerical solutions and the two asymptotic 
solutions as function of $t / x$. We plot on this figure $\bar{\tau}_{b} \sqrt{x}$ which is $\sqrt{x / \pi t}$ for small $t / x$ (Stokes solution) and becomes 0.322 (the Blasius value) for large $t / x$. On the solution of ESW, the unsteady part of ESW solution presents a small difference as well. This is simply due to the fact that the Blasius profile and the Stokes one have not exactly the shame shape as we have observed for $\delta_{1}$. The value $f_{2} H$ given by the Stokes solution is greater than that of the Blasius solution. Good agreement was found on the solution of MLSW: the solution goes from Stokes to Blasius behaviours; the transition between these two regimes is clearly captured.

\subsection{Flows over a small bump}

This final section considers cases with a flat bottom with a bump. The length of the bump is chosen to be compatible with the classical shallow water model. Hence, we consider now a domain $x \in[0,2]$ with a small bump of Gaussian form placed at the center:

$$
f_{b}=\alpha \mathrm{e}^{-\frac{(x-1)^{2}}{2 \sigma^{2}}}
$$

with $\alpha$ and $\sigma$ given. The domain was discretized with $\Delta x=10^{-3}$. Falkner-Skan closure, which includes as well the Blasius one, is used to compute the shape and friction factors. As defined by (3.17), we have to compute the pressure gradient parameter $\Lambda_{1}=\delta_{1}^{2} \partial_{x} u_{e}$ for which accurate approximation for the partial derivative $\partial_{x} u_{e}$ is required in order to provide a faithful representation of the shape of thin viscous layer. Therefore, we test second order and fourth-order finite difference derivative:

$$
\left(\partial_{x} u_{e}\right)_{j}=\frac{\left(u_{e}\right)_{j-2}-8\left(u_{e}\right)_{j-1}+8\left(u_{e}\right)_{j+1}-\left(u_{e}\right)_{j+2}}{12 \Delta x}+o\left(\Delta x^{4}\right) .
$$

Influence of the flow regime. We use first $\alpha=0.01$ and $\sigma=0.1$. As in the test case of an impulsively started flow, the displacement thickness develops from zero, at $t=0$. It reaches a steady value when the time is large enough, typically when $t>x H / u_{e} \simeq 6$ from characteristic solution (6.5) applied for this case. However, this steady solution is no longer Blasius but is slightly perturbed due to the presence of the bump; this perturbation depends furthermore on the flow regime, as one can observe on Figure 11.

For sub-critical case, the flow is accelerated on the upstream side of the bump while it is decelerated on the downstream side. As a consequence, the displacement thickness decreases before the crest and increases after. This behaviour is also well reported by classical shallow water model, even with linearized solution (2.22). However, ESW provides an asymmetric friction due to inertial effects of the fluid, compared with (2.22). More precisely, the friction reaches its maximum before the crest for sub-critical flow while for super-critical case, it becomes maximum after the crest, see Figure 11 (right plot). This is in fact the important phase-lag behaviours that we have found to cope with ESW.

Phase-lag reduced for shorter bump. Here we investigate the influence of the length of the bump on the friction computed with ESW model. Returning to sub-critical case, we perform now the same test case but with a shorter bump, by imposing $\sigma=0.05$. Regarding the result given with Falkner-Skan closure, i.e. when the shape factor depends on velocity gradient $\partial_{x} u_{e}$, Figure 12 (left) shows that the variation of friction is more important than the case with $\sigma=0.1$. The phase-lag is always observed.

To highlight the role of the closure imposed in viscous layer, we do again this test but with a constant value of shape factor (e.g. by using Blasius closure). It can be observed on Figure 12 (right) that both amplitude and phase-lag of friction are significantly reduced compared to the case with (variable) Falkner-Skan closure. Moreover, constant shape factor does not allow the friction to decrease enough in decelerated region localized at downstream side of the bump. As a consequence, this kind of closure is unable to recover reverse flow whatever the bump shape.

Larger friction for higher bump, reverse flow observation. This last test case is devoted to highlight the possibility of ESW to capture indeed reverse flow if the bump is high enough. We fix the bump length to be $\sigma=0.1$ and change $\alpha$. Falkner-Skan closure (3.17) is of course imposed. Figure 13 shows the displacement 

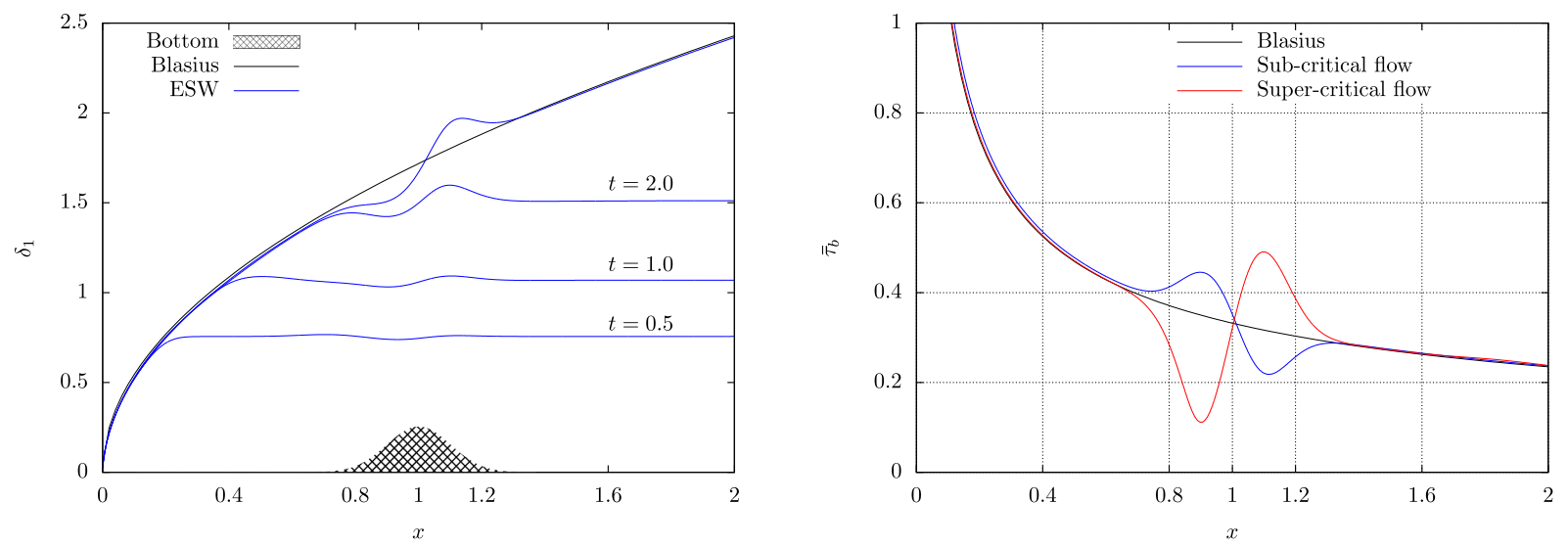

FiguRE 11. Flow over a small bump at different times. Left panel: development of displacement thickness at different times in sub-critical case. Right panel: behaviours of friction in sub-critical and super-critical cases at long time $(t=6)$.
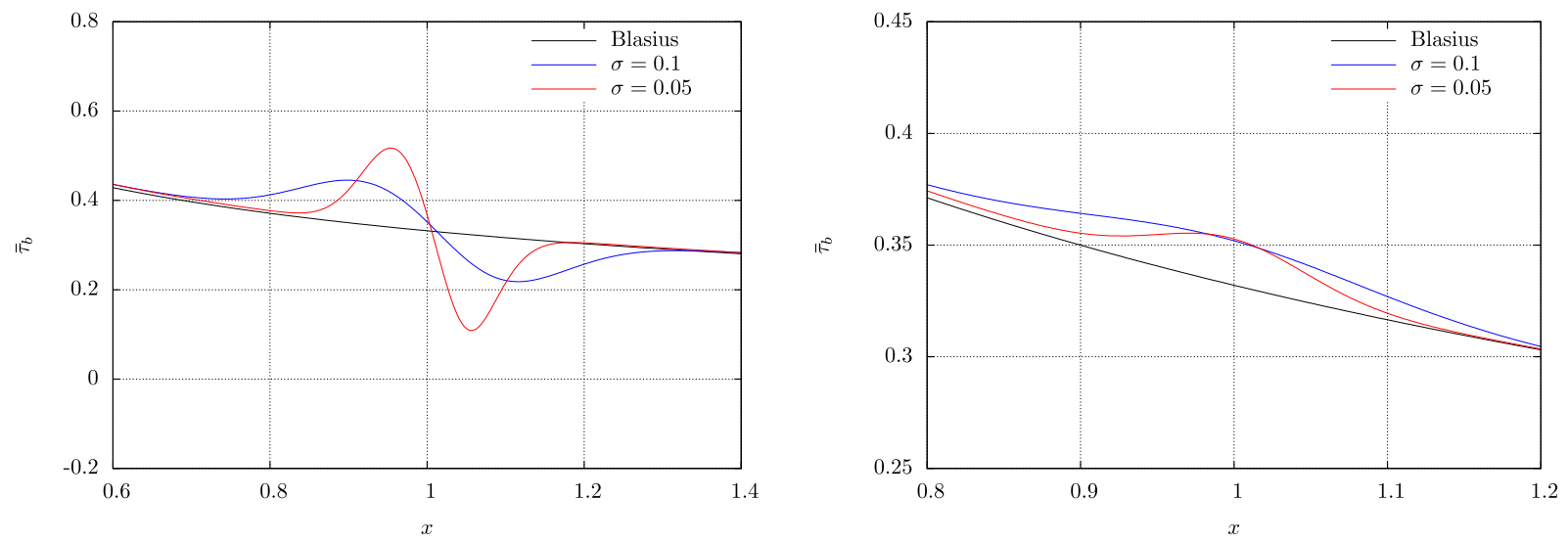

Figure 12. Phase-lag for two short bumps $(\alpha=0.05)$ compared to Blasius solution $(\alpha=0.1)$. Left panel: friction with Falkner-Skan closure (3.17). Right panel: friction with constant value of $H=2.59$, and $f_{2}=0.22$ from Blasius solution.

thickness and the friction for increasing $\alpha$ up to negative value of friction (which defines steady boundary layer separation). Associated to boundary layer separation is an increased displacement thickness.

Note that accurate estimation of velocity gradient $\partial_{x} u_{e}$ is very important to capture this particular phenomena. That is why we have used the fourth-order formula (6.6). Using a second-order approximation for $\partial_{x} u_{e}$ results just in the incipient separation.

Finally, performing this test case with the MLSW model allows to find as well the phase-lag, but the amplitude of the friction is smaller as observed on Figure 14. It seems that the numerical solver of the MLSW model together with the first-order approximation (5.19) on $\bar{\tau}_{b}$, and even with 100 layers, is not enough to accurately capture the friction.

Nevertheless, plotting the friction factor $f_{2}$ as a function of the shape factor $H$ and comparing it with FalknerSkan closure (3.17) shows that indeed, the reduced shear and shape factors computed from the MLSW profiles 

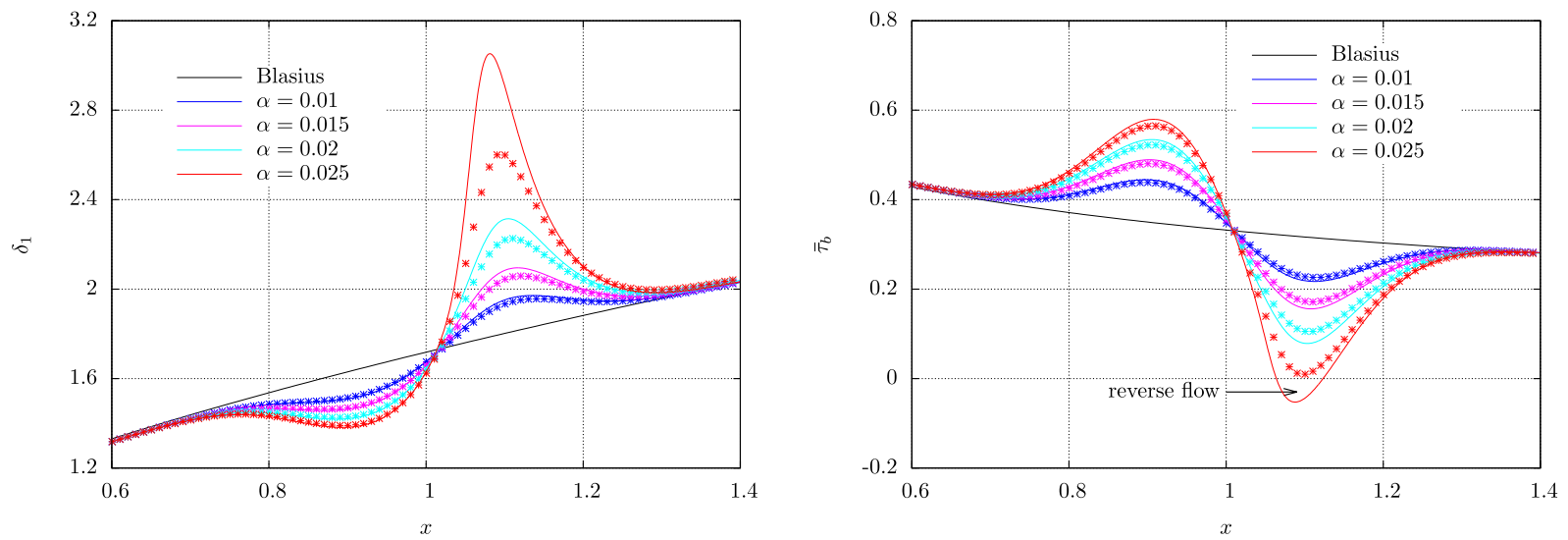

FIGURE 13. ESW for various bump height $\alpha$ at fixed lenght $\sigma=0.1$ with 2nd order (symbols) and 4th order (lines) discretization. Left panel: displacement thickness. Right panel: wall shear stress. The influence of the 2 nd and 4 th order derivative is noticeable for $\alpha=0.025$ near separation.
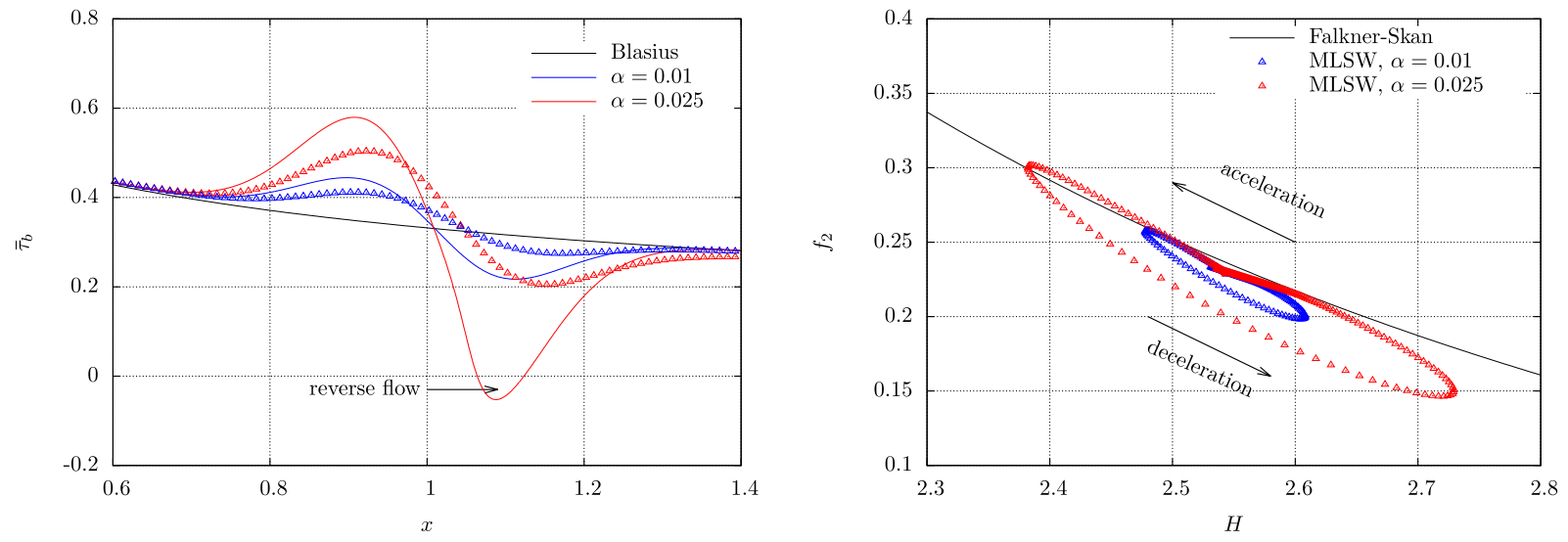

FiguRE 14. Friction of ESW (lines) vs. MLSW (symbols) for two values of $\alpha$. Right panel: relation between $H$ and $f_{2}$ given by MLSW (symbols) compared to Falkner-Skan closure used for ESW (line).

are close to Falkner Skan curve. The smaller the angle, the closer the curves. The agreement is better during the accelerated phase of the flow.

\section{Conclusion}

In order to improve shallow water models, this paper proposed a novel description of parietal friction for free surface shallow flows (long wave approximation) in large Reynolds number limit. The proposed model relies on a perfect fluid - viscous layer decomposition. It consists in a system of four equations: conservation of mass (2.14), momentum (4.6), an inviscid fluid equation (4.7), and the von Kármán equation (4.8). The first two are similar to classical shallow water system with a slight correction on momentum flux, and with a specific friction term. 
Two equivalent versions of the model can be obtained by interpreting it from two points of view. In the first one, the viscous layer acts as a new topography for the model, see (4.9). In the second one, this is an example of "Interactive Boundary Layer" or "Viscous Inviscid Interaction", see (4.11), and it seems to be the most convenient choice for numerical purposes. In these models the friction term is no longer an empirical combination of velocity and depth (as in usual laws such as Darcy or Manning) but the result of a viscous layer approach. A crucial point at this stage is a choice of an appropriate closure for the shape factor and the friction factor in the viscous layer, in order to obtain a closed system after integration.

As it is, the friction term actually depends on the topography, as evidenced by the examples provided in the last part of this work. In particular its maximum is reached before or after the summit of a bump, depending on the criticity of the flow. Also, possible boundary layer separation with recirculation downstream (in subcritical case) of the bump can be observed as in similar cases in litterature ([40-42] in case of flows in pipes, and as in preliminary comparisons with multilayer shallow water [2]). These two behaviors are impossible to observe in the classical Shallow Water model.

The approach we propose is presently restricted to laminar flows, but the ideas developed here can be extended with little modifications to turbulent profiles. The main drawback of this viscous layer/inviscid fluid decomposition, is that it forbids the viscous layer to fill the whole water depth far downstream of the bump, and wet/dry transitions as well. Extra modelling in this direction, as well as a more careful study of the closure laws in the viscous layer have now to be worked out and tested. Also, the numerical study has to be improved, with focus on the boundary conditions and the nonconservative product. In spite of these limitations, the model is a good compromise between the classical shallow water system and RNSP equations (discretized with the Multilayer Saint-Venant scheme [2]), much more expensive in time computation.

Although the present closure is mathematically derived under a fixed bottom hypothesis, the model could still be applicable for the case of slowly moving bed due to erosion, e.g. the bedload case in alluvial channel. Indeed, in many river applications, the time scale of erosion is much slower than that of flow. Accordingly, hydrodynamic and morphodynamic solvers are often decoupled meaning that the bottom remains unchanged while solving the flow. Even in such consideration, the phase-lag behaviour is still important to preserve physical instability of erodible bed - such as dune and ripple formation - so the proposed model is relevant. Studying an adequate closure law for the case of (rapid) moving bed is needed in further work because the main objective of the model is to improve erosion modelling.

\section{Appendix A. Proof of Proposition 4.1}

We start from the von Kármán equation and introduce (3.8) to obtain

$$
\partial_{t}\left(h u_{e}\right)-\partial_{t}(h U)+\left(h u_{e}-U\right) \partial_{x} u_{e}+\bar{\delta} \partial_{x}\left(u_{e}^{2} \delta_{2}\right)=\bar{\delta} \bar{\tau}_{b} .
$$

To this equation we add (4.1), the parietal term disappears, leading to

$$
\partial_{t}\left(h u_{e}\right)+\partial_{x} J+h u_{e} \partial_{x} u_{e}-h U \partial_{x} u_{e}+\bar{\delta} \partial_{x}\left(u_{e}^{2} \delta_{2}\right)=-h \partial_{x} p .
$$

Developing the time derivative and simplifying with (2.11) we obtain

$$
u_{e} \partial_{t} h+\partial_{x} J-h U \partial_{x} u_{e}+\partial_{x}\left(\bar{\delta} u_{e}^{2} \delta_{2}\right)=0
$$

Finally, we use (2.14) to eliminate the time derivative, regroup terms and get

$$
\partial_{x}\left(J-h u_{e} U+\bar{\delta} \delta_{2} u_{e}^{2}\right)=0,
$$

so that, up to a constant which can be taken equal to zero by considering that the flux is zero when the velocity is zero, we have

$$
J=h u_{e} U-\bar{\delta} \delta_{2} u_{e}^{2}
$$


which together with (3.8) gives precisely (4.2).

Conversely, we consider (3.8) and use successfully the mass and momentum balance equations

$$
\begin{aligned}
\partial_{t}\left(u_{e} \bar{\delta} \delta_{1}\right) & =\partial_{t}\left(h u_{e}\right)-\partial_{t}(h U)=u_{e} \partial_{t} h+h \partial_{t} u_{e}+\partial_{x}\left(\left(h-\bar{\delta}\left(\delta_{1}+\delta_{2}\right)\right) u_{e}^{2}\right)+h \partial_{x} p+\bar{\delta} \bar{\tau}_{b} \\
& =-u_{e} \partial_{x}(h U)-h u_{e} \partial_{x} u_{e}-h \partial_{x} p+\partial_{x}\left(h u_{e}^{2}\right)-\bar{\delta} \partial_{x}\left(\left(\delta_{1}+\delta_{2}\right) u_{e}^{2}\right)+h \partial_{x} p+\bar{\delta} \bar{\tau}_{b} \\
& =-u_{e} \partial_{x}\left(h u_{e}\right)+u_{e} \partial_{x}\left(\bar{\delta} \delta_{1} u_{e}\right)-h u_{e} \partial_{x} u_{e}+\partial_{x}\left(h u_{e}^{2}\right)-\bar{\delta} \partial_{x}\left(\left(\delta_{1}+\delta_{2}\right) u_{e}^{2}\right)+\bar{\delta} \bar{\tau}_{b} \\
& =-\bar{\delta}\left(-u_{e} \partial_{x}\left(\delta_{1} u_{e}\right)+\partial_{x}\left(\left(\delta_{1}+\delta_{2}\right) u_{e}^{2}\right)-\bar{\tau}_{b}\right) .
\end{aligned}
$$

Noting that $\partial_{x}\left(\delta_{1} u_{e}^{2}\right)=u_{e} \partial_{x}\left(\delta_{1} u_{e}\right)+\delta_{1} u_{e} \partial_{x} u_{e}$ we recover as required the von Kármán equation.

\section{Appendix B. Proof of Proposition 4.2}

From (3.8)

$$
\begin{aligned}
\partial_{t}\left(u_{e} \bar{\delta} \delta_{1}^{*}\right) & =\partial_{t}\left(h u_{e}\right)-\partial_{t}(h U)=u_{e} \partial_{t} h+h \partial_{t} u_{e}+\partial_{x}\left(\left(h-\bar{\delta}\left(\delta_{1}+\delta_{2}\right)\right) u_{e}^{2}\right)+h \partial_{x} p+\bar{\delta} \bar{\tau}_{b} \\
& =-u_{e} \partial_{x}(h U)-h u_{e} \partial_{x} u_{e}-h \partial_{x} p+\partial_{x}\left(h u_{e}^{2}\right)-\bar{\delta} \partial_{x}\left(\left(\delta_{1}+\delta_{2}\right) u_{e}^{2}\right)+h \partial_{x} p+\bar{\delta} \bar{\tau}_{b} \\
& =-u_{e} \partial_{x}\left(h u_{e}\right)+u_{e} \partial_{x}\left(\bar{\delta} \delta_{1}^{*} u_{e}\right)-h u_{e} \partial_{x} u_{e}+\partial_{x}\left(h u_{e}^{2}\right)-\bar{\delta} \partial_{x}\left(\left(\delta_{1}+\delta_{2}\right) u_{e}^{2}\right)+\bar{\delta} \bar{\tau}_{b} \\
& =-\bar{\delta}\left(-u_{e} \partial_{x}\left(\delta_{1}^{*} u_{e}\right)+\partial_{x}\left(\left(\delta_{1}+\delta_{2}\right) u_{e}^{2}\right)-\bar{\tau}_{b}\right)
\end{aligned}
$$

Now using the von Kármán equation to eliminate $\delta_{2}$, we obtain

$$
\begin{aligned}
\bar{\delta} \partial_{t}\left(u_{e} \delta_{1}^{*}\right) & =-\bar{\delta}\left(-u_{e} \partial_{x}\left(\delta_{1}^{*} u_{e}\right)+\partial_{x}\left(\delta_{1} u_{e}^{2}\right)-\partial_{t}\left(u_{e} \delta_{1}\right)-u_{e} \delta_{1} \partial_{x} u_{e}\right) \\
& =-\bar{\delta}\left(-u_{e} \partial_{x}\left(u_{e}\left(\delta_{1}^{*}-\delta_{1}\right)\right)-\partial_{t}\left(u_{e} \delta_{1}\right)\right),
\end{aligned}
$$

which is the desired result.

Acknowledgements. We would like to thank Nicole Goutal (EDF R\&D, Laboratoire d'Hydraulique Saint-Venant) for insightful discussions.

\section{REFERENCES}

[1] F. Alcrudo and F. Benkhaldoun, Exact solutions to the Riemann problem of the shallow water equations with a bottom step. Comput. Fluids 30 (2001) 643-671.

[2] E. Audusse, M.-O. Bristeau, B. Perthame and J. Sainte-Marie, A multilayer Saint-Venant system with mass exchanges for shallow water flows. Derivation and numerical validation. ESAIM: M2AN 45 (2011) 169-200.

[3] J. Best, The fluid dynamics of river dunes: a review and some future research directions. J. Geophys. Res. Earth Surf. 110 (2005) F4.

[4] F. Bouchut, Nonlinear stability of finite volume methods for hyperbolic conservation laws, and well-balanced schemes for sources. Frontiers in Mathematics Birkhäuser Verlag, Basel 2004 (2004).

[5] F. Bouchut, J. Le Sommer and V. Zeitlin, Frontal geostrophic adjustment and nonlinear wave phenomena in one-dimensional rotating shallow water. Part 2. High-resolution numerical simulations. J. Fluid Mech. 514 (2004) 35-63.

[6] J. Burguete and P. Garcì-Navarro, Implicit schemes with large time step for non-linear equations: application to river flow hydraulics. Int. J. Numer. Meth. Fluids 46 (2004) 607-636.

[7] V. Caleffi, A. Valiani and A. Zanni, Finite volume method for simulating extreme flood events in natural channels. J. Hydraul. Res. 41 (2003) 167-177.

[8] M.J.C. Díaz, E.D. Fernández-Nieto, A.M. Ferreiro, Sediment transport models in shallow water equations and numerical approach by high order finite volume methods. Comput. Fluids 37 (2008) 299-316.

[9] T. Cebeci and H.B. Keller, Shooting and parallel shooting methods for solving the falkner-skan boundary-layer equation. $J$. Comput. Phys. 7 (1971) 289-300.

[10] F. Charru, B. Andreotti and P. Claudin, Sand ripples and dunes. Annu. Rev. Fluid Mech. 45 (2013) $469-493$.

[11] V.T. Chow, Open-Channel Hydraulics. McGraw-Hill, New York, NY (1959).

[12] A.J.-C. de Saint Venant, Théorie du mouvement non-permanent des eaux, avec application aux crues des rivières et à l'introduction des marées dans leur lit. C. R. Acad. Sci. 73 (1871) 147-154. 
[13] G. Dal Maso, P. LeFloch and F. Murat, Definition and weak stability of nonconservative products. J. Math. Pures Appl. 74 (1995) 483-548.

[14] O. Delestre, S. Cordier, F. James and F. Darboux, Simulation of rain-water overland-flow. In: Proceedings of the 12th International Conference on Hyperbolic Problems. University of Maryland, College Park (USA) (2008).

[15] E. Tadmor, J.-G. Liu and A. Tzavaras Eds., Proceedings of symposia in applied mathematics. Amer. Math. Soc. 67 (2009) 537-546.

[16] A. Doré, P. Bonneton, V. Marieu and T. Garlan, Numerical modeling of subaqueous sand dune morphodynamics. J. Geophys. Res. Earth Surf. 121 (2016) 565-587.

[17] A. Ellis and A. Fowler, On an evolution equation for sand dunes. SIAM J. Appl. Math. (2010).

[18] M. Esteves, X. Faucher, S. Galle and M. Vauclin, Overland flow and infiltration modelling for small plots during unsteady rain: numerical results versus observed values. J. Hydrol. 228 (2000) 265-282.

[19] F.M. Exner, Uber die wechselwirkung zwischen wasser und geschiebe in flussen. Akad. Wiss. Wien Math. Naturwiss. Klasse 134 (1925) 165-204.

[20] V. Falkner and S.W. Skan, Solutions of the boundary-layer equations. London, Edinburgh, and Dublin Philos. Mag. J. Sci. 12 (1931) 865-896.

[21] A. Fowler, Dunes and drumlins. In: Geomorphological Fluid Mechanics. Springer, Berlin (2001) 430-454.

[22] D.L. George, Finite volume methods and adaptive refinement for tsunami propagation and inundation. Ph.D. thesis, University of Washington (2006).

[23] J.-F. Gerbeau and B. Perthame, Derivation of viscous Saint-Venant system for laminar shallow water; numerical validation. Discrete Contin. Dyn. Syst. Ser. B 1 (2001) 89-102.

[24] A. Ghigo, J.-M. Fullana and P.-Y. Lagrée, A 2D nonlinear multiring model for blood flow in large elastic arteries. J. Comput. Phys. 350 (2017) 136-165.

[25] E. Godlewski and P.-A. Raviart, Numerical approximations of hyperbolic systems of conservation laws. Applied Mathematical Sciences. Springer-Verlag, New York, NY 118 (1996).

[26] N. Goutal, M.-H. Le and P. Ung, A Godunov-type scheme for shallow water equations dedicated to simulations of overland flows on steep slopes. In: International Conference on Finite Volumes for Complex Applications. Springer, Berlin (2017) $275-283$.

[27] N. Goutal and F. Maurel, Proceedings of the 2nd workshop on dam-break wave simulation. Technical Report, EDF-DER (1997).

[28] R.S. Govindaraju, Modeling overland flow contamination by chemicals mixed in shallow soil horizons under variable source area hydrology. Water Resour. Res. 32 (1996) 753-758.

[29] A. Harten, P.D. Lax and B. van Leer, On upstream differencing and Godunov-type schemes for hyperbolic conservation laws. SIAM Rev. 25 (1983) 35-61.

[30] A.J. Hogg and D. Pritchard, The effects of hydraulic resistance on dam-break and other shallow inertial flows. J. Fluid Mech. 501 (2004) 179-212.

[31] R.S. Johnson, A Modern Introduction to the Mathematical Theory of Water Waves. Cambridge University Press, Cambridge (1997).

[32] S. Kalliadasis, C. Ruyer-Quil, B. Scheid and M.G. Velarde, Falling Liquid Films. Springer Science \& Business Media 176 (2011).

[33] J.F. Kennedy, The mechanics of dunes and antidunes in erodible-bed channels. J. Fluid Mech. 16 (1963) 521-544.

[34] M.M. Keshtkar and M. Ezatabadi, Numerical solution for the falkner-skan boundary layer viscous flow over a wedge. Int. J. Eng. Sci. Technol. 3 (2013) 18-36.

[35] D.-H. Kim, Y.-S. Cho and Y.-K. Yim, Propagation and run-up of nearshore tsunamis with hllc approximate riemann solver. Ocean Eng. 34 (2007) 1164-1173.

[36] G. Kirstetter J. Hu, O. Delestre, F. Darboux, P.-Y. Lagrée, S. Popinet, J.M. Fullana, C. Josserand, Modeling rain-driven overland flow: empirical versus analytical friction terms in the shallow water approximation. J. Hydrol. 536 (2016) 1-9.

[37] K.K.J. Kouakou and P.-Y. Lagrée, Evolution of a model dune in a shear flow. Eur. J. Mech.-B/Fluids 25 (2006) 348-359.

[38] P.-Y. Lagrée, A triple deck model of ripple formation and evolution. Phys. Fluids 15 (2003) 2355-2368.

[39] P.-Y. Lagrée, Interactive boundary layer (IBL). In: Asymptotic Methods in Fluid Mechanics: Survey and Recent Advances. Springer, Berlin (2010) 247-286.

[40] P.-Y. Lagrée, E. Berger, M. Deverge, C. Vilain and A. Hirschberg, Characterization of the pressure drop in a 2D symmetrical pipe: some asymptotical, numerical, and experimental comparisons. ZAMM-J. Appl. Math. Mech./Z. Angew. Math. Mech. 85 (2005) 141-146.

[41] P.-Y. Lagrée and S. Lorthois, The RNS/Prandtl equations and their link with other asymptotic descriptions: application to the wall shear stress scaling in a constricted pipe. Int. J. Eng. Sci. 43 (2005) 352-378.

[42] P.-Y. Lagrée, A. Van Hirtum and X. Pelorson, Asymmetrical effects in a 2D stenosis. Eur. J. Mech.-B/Fluids 26 (2007) 83-92.

[43] M.-H. Le, S. Cordier, C. Lucas and O. Cerdan, A faster numerical scheme for a coupled system modeling soil erosion and sediment transport. Water Resour. Res. 51 (2015) 987-1005.

[44] M.-R. Muñoz-Ruiz and C. and Parés, Godunov method for nonconservative hyperbolic systems. ESAIM: M2AN 41 (2007) $169-185$

[45] M. Nabi, H. Vriend, E. Mosselman, C. Sloff and Y. Shimizu, Detailed simulation of morphodynamics: 3. Ripples and dunes. Water Resour. Res. 49 (2013) 5930-5943. 
[46] S. Naqshband, O. Duin, J. Ribberink and S. Hulscher, Modeling river dune development and dune transition to upper stage plane bed. Earth Surf. Process. Landf. 15 (2015) 323-335.

[47] R. Nickalls, 95.60 a new bound for polynomials when all the roots are real. Math. Gazette 95 (2011) 520-526.

[48] A.J. Paarlberg, C.M. Dohmen-Janssen, S.J. Hulscher and P. Termes, Modeling river dune evolution using a parameterization of flow separation. J. Geophys. Res. Earth Surf. 114 (2009) F1.

[49] S. Popinet, Quadtree-adaptative tsunami modelling. Ocean Dyn. 61 (2011) 1261-1285.

[50] L. Prandtl, Motion of fluids with very little viscosity. NACA Transl. 452 (1928) 1-8.

[51] G.L. Richard and S.L. Gavrilyuk, A new model of roll waves: comparison with brock's experiments. J. Fluid Mech. 698 (2012) $374-405$.

[52] J. Rivlin (Byk) and R. Wallach, An analytical solution for the lateral transport of dissolved chemicals in overland flow. Water Resour. Res. 31 (1995) 1031-1040.

[53] H. Schlichting, Boundary-layer Theory. McGraw-Hill, New York, NY (1968).

[54] K. Stewartson, On the impulsive motion of a flat plate in a viscous fluid. Quart. J. Mech. Appl. Math. 4 (1951) $182-198$.

[55] K. Stewartson, On the impulsive motion of a flat plate in a viscous fluid. II. Quart. J. Mech. Appl. Math. 26 (1973) $143-152$.

[56] L. Tatard, O. Planchon, J. Wainwright, G. Nord, D. Favis-Mortlock, N. Silvera, O. Ribolzi, M. Esteves and C.-H. Huang, Measurement and modelling of high-resolution flow-velocity data under simulated rainfall on a low-slope sandy soil. J. Hydrol. 348 (2008) 1-12.

[57] A. Valiani, V. Caleffi and A. Zanni, Case study: malpasset dam-break simulation using a two-dimensional finite volume methods. J. Hydraul. Eng. 128 (2002) 460-472.

[58] M. Van Dyke, Perturbation Methods in Fluid Mechanics. Parabolic Press, Incorporated, Hackensack, NY (1975).

[59] A.I. Vol'pert, The spaces BV and quasilinear equations. Matematicheskii Sbornik 115 (1967) 255-302.

[60] J. Zhang and B. Chen, An iterative method for solving the falkner-skan equation. Appl. Math. Comput. 210 (2009) $215-222$. 